\title{
Density, Sound Speed and Derived Thermophysical Properties of $n$-Nonane at Temperatures Between (283.15 and 473.15) K and at Pressures up to $390 \mathrm{MPa}$
}

\author{
Weparn J Tay and J P Martin Trusler \\ Department of Chemical Engineering, Imperial College London, South Kensington Campus, \\ London SW7 2AZ
}

\begin{abstract}
In this paper, we present density and speed-of-sound experimental measurements for $n$ nonane at temperatures between (283.15 and 473.15) $\mathrm{K}$ and pressures up to $68 \mathrm{MPa}$ and 390 $\mathrm{MPa}$ respectively. The density measurements were performed with a vibrating-tube densimeter and the speed-of-sound measurements were carried out in a dual-path pulse-echo apparatus. The vibrating-tube densimeter was calibrated using pure helium and water over the full range of temperature and pressure investigated, while the speed-of-sound apparatus was calibrated using pure water at low pressure over the full range of temperature. The expanded relative uncertainties of the measurements were $0.08 \%$ for density and between (0.1 and 0.3 ) \% for sound speed at $95 \%$ confidence. The density data were correlated with the modified Tait equation over the entire temperature and pressure range, with an absolute average relative deviation of $0.006 \%$. An empirical equation was developed to represent the sound speed data with an absolute average relative deviation of $0.03 \%$. Both sets of data were compared with the predictions from the equation of state developed by Lemmon and Span. Comparisons have also been made with the available literature and satisfactory agreement was found. Correlations were developed for the density and isobaric heat capacity of the liquid as functions of temperature at a reference pressure of $0.1 \mathrm{MPa}$, the latter based on literature data. Combining these correlations with the sound-speed surface, properties of the liquid were computed by thermodynamic integration up to a pressure of $390 \mathrm{MPa}$. Density, isobaric heat capacity, isothermal compressibility and isobaric expansivity values are reported, and their uncertainties were carefully investigated.
\end{abstract}

Keywords: Compressibility; Density; Expansivity; Heat capacity; $n$-Nonane; Speed of sound. 


\section{Introduction}

Thermophysical properties of hydrocarbons are of interest in many industries, especially in the oil and gas and chemical sectors. They play an important role in the design of safe and efficient operations and often dominate in determining the economics of a process [1]. Alkanes are often the most abundant components in crude oils and during processes such as oil extraction and enhanced oil recovery (EOR) may be subject to high-temperature and highpressure (HTHP) conditions with, for example, temperatures up to $450 \mathrm{~K}$ and pressures up to $110 \mathrm{MPa}$ in a hyperbaric oil reservoir [2; 3]. In order to predict the behaviour of crude oil during these processes, it is essential to study the thermophysical properties of hydrocarbons for characterisation purposes. The oil and gas industry currently relies on simple models, such as cubic equations of state (EoS), to predict the thermodynamic properties of hydrocarbons at extreme conditions [4; 5]. Unfortunately, these models are not very accurate and more sophisticated models are required to predict properties such as density, compressibility and expansivity under HTHP conditions. However, the development of such models requires experimental data.

Although the thermodynamic properties of hydrocarbons have been studied by many authors, the available data are quite limited at HTHP conditions, even for simple alkane systems. Comparing even and odd carbon numbers, it is found that data for the alkanes with odd carbon numbers are especially scarce under HTHP conditions. To help fill this gap, the present study focuses on the $9^{\text {th }}$ normal alkane, $n$-nonane, with the objective of deriving a comprehensive set of thermodynamic data over wide ranges of temperature and pressure in the liquid region. The choice of $n$-nonane for the present study was reinforced by the recommendations reported with the GERG-2008 EoS [6] which included new measurements of the thermodynamic properties for $n$-nonane over wide ranges of temperatures and pressures.

The most accurate wide-range EoS currently available of $n$-nonane was developed by Lemmon and Span in 2006 [7]. This model is valid from the triple-point temperature of $219.7 \mathrm{~K}$ to $575 \mathrm{~K}$ and at pressures up to $800 \mathrm{MPa}$. The stated relative uncertainties for liquid-phase density are $0.1 \%$ for saturated states between (280 and 335) K, $0.2 \%$ for other liquid states at $p \leq 10 \mathrm{MPa}, 0.3 \%$ for pressures between (10 and 100) MPa and $0.5 \%$ at higher pressures. Relative uncertainties for other properties of the compressed liquid were given as $2 \%$ for isobaric heat capacity at $T \leq 550 \mathrm{~K}$ and $1 \%$ for speed of sound at $T \leq 430 \mathrm{~K}$; beyond these temperatures the EoS was not validated by any experimental data. 
TABLE 1. Summary of available literature data for the thermodynamic properties of compressed liquid $n$-nonane where $\rho$ is density, $v_{\mathrm{R}}$ is relative volume, $v$ is specific volume, $c_{p}$ is isobaric specific heat capacity, $c$ is speed of sound, $T$ is temperature, $p$ is pressure and $\Delta_{\text {AARD }}$ is absolute average relative deviation from the equation of state of Lemmon and Span [7].

\begin{tabular}{|c|c|c|c|c|c|}
\hline Property & Method & $T / K$ & $p / \mathrm{MPa}$ & $\Delta_{\text {AARD }}$ & Reference \\
\hline$\rho$ & Piezometer & 311 to 511 & 0.2 to 69 & $0.28 \%$ & Carmichael et al. [8] \\
\hline$v_{\mathrm{R}}$ & Bellows & 303 to 393 & 0.1 to 118 & $0.11 \%$ & Boelhouwer [9] \\
\hline$v$ & Piezometer & 303 to 573 & 5.0 to 500 & $0.27 \%$ & Doolittle et al. [10] \\
\hline$\rho$ & Piezometer & 303 to 423 & 0.1 to 800 & $0.19 \%$ & Grindley and Lind [11] \\
\hline$\rho$ & Bellows & 248 to 373 & 0.1 to 301 & $0.17 \%$ & Scaife and Lyons [12] \\
\hline$\rho$ & Vibrating tube & 313 to 373 & 0.1 to 10 & $0.07 \%$ & Banipal et al. [13] \\
\hline$\rho$ & Piezometer & 303 to 363 & 0.1 to 800 & $0.23 \%$ & Brostow et al. [14] \\
\hline$\rho$ & Vibrating tube & 283 to 318 & 0.1 to 60 & $0.10 \%$ & Valencia et al. [15] \\
\hline$\rho$ & Vibrating tube & 298 to 328 & 0.1 to 40 & $0.11 \%$ & Moravkova et al. [16] \\
\hline$\rho$ & Single-sinker densimeter & 233 to 373 & 0.1 to 30 & $0.07 \%$ & Schilling et al. [17] \\
\hline$c_{p}$ & Flow calorimeter & 323 to 590 & 5 to 60 & $0.50 \%$ & Kuznetsov et al.[18] \\
\hline$c_{p}$ & DSC $^{a}$ & 318 to 373 & 0.1 to 10 & $0.47 \%$ & Banipal et al. [13] \\
\hline$c$ & Single-path pulse-echo & 253 to 412 & 0.1 to 140 & $0.40 \%$ & Boelhouwer [19] \\
\hline$c$ & Single-path pulse-echo & 303 to 393 & 0.1 to 118 & $0.35 \%$ & Badalyan et al.[20] \\
\hline$c$ & Single-path pulse-echo & 303 to 433 & 10 to 600 & $1.39 \%$ & Zotov et al. [21] \\
\hline$c$ & Double-path pulse- echo & 293 to 393 & 0.1 to 100 & $0.27 \%$ & Lago et al. [22] \\
\hline
\end{tabular}

a DSC = differential scanning calorimetry

The available experimental data for thermodynamic properties of compressed liquid $n$-nonane are summarised in Table 1 and include volumetric properties $[8 ; 9 ; 10 ; 11 ; 12 ; 13 ; 14 ; 15 ; 16$; $17]$, isobaric heat capacity $[13 ; 18]$ and speed of sound $[19 ; 20 ; 21 ; 22]$. The absolute average relative deviation $\Delta_{\mathrm{AARD}}$ from the EoS of Lemmon and Span is also given for each data set. Lemmon and Span noted that there were inconsistencies between the volumetric data of Scaife and Lyons [12] and Boelhouwer [9], on the one hand, and Grindley and Lind [11] and Doolittle [10], on the other hand. The data of Schilling et al. [17] are characterised by the smallest expanded relative uncertainty, $0.02 \%(k=2)$, and were intended to enable the use 
of $n$-nonane as a calibrating fluid for industrial densimeters such as vibrating-tube densimeters. The two available literature sources for isobaric heat capacity are in good agreement with the equation of state, as are three of the four sources for sound-speed. The speed of sound has been well characterised at temperatures between (253 and 433) K and at pressures up to $600 \mathrm{MPa}$; beyond these ranges, no data are available. The most recent sound-speed data are those of Lago et al. [22], who used the double-path pulse-echo technique at temperatures between $(293.15$ and 393.15$) \mathrm{K}$ and pressures up to $100 \mathrm{MPa}$, and claim a relative expanded uncertainty of $0.2 \%(k=2)$. Boelhouwer [19] made measurements using a single-path pulse-echo apparatus with a claimed expanded relative uncertainty of 0.2 $\%$. Badalyan et al. [20] used a similar technique at pressures up to $118 \mathrm{MPa}$ and reported an expanded uncertainty of $2 \mathrm{~m} \cdot \mathrm{s}^{-1}$ (about $0.15 \%$ at the median sound speed). Zotov et al. [21] report results over a wide temperature range with pressures up to $600 \mathrm{MPa}$ and claim an expanded relative uncertainty of $0.3 \%$. In the overlapping pressure ranges, these data agree well with the other three sources and with the EoS but deviations from the EoS of increasing magnitude are observed at higher pressures. In addition to these sources, numerous authors (including, for example, Casas et al. [23], Pereira et al. [24] and Aminabhavi and Gopallkrishna [25]) have reported speeds of sound measured in $n$-nonane at pressures near to $0.1 \mathrm{MPa}$.

TABLE 2. Summary of available literature data for the isobaric specific heat capacity $c_{p}$ of liquid $n$-nonane at $p \leq 0.1 \mathrm{MPa}$ and $T \geq 273.15 \mathrm{~K}$. Also given is the absolute average relative deviation $\Delta_{\text {AARD }}$ from the equation of state of Lemmon and Span [7].

\begin{tabular}{cccc}
\hline Method & T/K & \multicolumn{1}{c}{$\Delta_{\text {AARD }}$} & Reference \\
\hline Picker flow calorimeter & 298.15 & $0.61 \%$ & Andreoli-Ball et al. [26] \\
Picker flow calorimeter & 298.15 & $0.36 \%$ & Grolier et al. [27] \\
Picker flow calorimeter & 298.15 & $0.08 \%$ & Calvo et al. [28] \\
DSC & 285 to 313 & $0.30 \%$ & Lei et al. [29] \\
DSC & 288 to 308 & $0.49 \%$ & Tovar et al. [30] \\
Adiabatic calorimeter & 274 to 313 & $0.56 \%$ & Finke et al. [31] \\
DSC & 288 to 308 & $0.46 \%$ & Pardo et al. [32] \\
Picker flow calorimeter & 298.15 & $0.26 \%$ & Wilhelm et al. [33] \\
Adiabatic calorimeter & 278 to 318 & $0.35 \%$ & Osborne \& Ginnings [34] \\
\hline
\end{tabular}


The objective of the present study was to provide new experimental data of low uncertainty for both density and sound speed, extending over wide ranges of temperature and pressure. Specifically, measurements were made at temperatures from (283.15 to 473.15 ) K, with pressures up to $65 \mathrm{MPa}$ for density and $390 \mathrm{MPa}$ for sound speed. We use thermodynamic integration to obtain a full description of the thermodynamic properties of liquid $n$-nonane over the whole region in which the speed of sound has been measured. Since this analysis requires as an input values of the isobaric specific heat capacity along a low-pressure isobar, we also review briefly the available literature data for this property. Table 2 details the available literature pertaining to pressures of $0.1 \mathrm{MPa}$ or below and to temperatures from $273.15 \mathrm{~K}$ upwards. Nine literature sources were identified which together cover temperatures from $274 \mathrm{~K}$ to $318 \mathrm{~K}$. As discussed further below, low pressure $c_{p}$ values may also be extracted from the data of Banipal et al. [13] and Kuznetsov et al. [18] by means of extrapolation, thereby extending the available data to higher temperatures.

\section{Materials and Methods}

\subsection{Materials}

The materials used are detailed in Table 3. The purity of the $n$-nonane was determined by the supplier to be mass fraction $w=0.998$ using gas chromatography with a flame ionisation detector. Specific impurities were not identified but, for purposes of uncertainty analysis, the impurities are assumed to be $\mathrm{C}_{8}$ or $\mathrm{C}_{10}$ paraffins. The purity of the methylbenzene was also analysed by the supplier and found to be $w=0.9996$. Gases were used without further purification. Liquid samples were degassed under vacuum with stirring immediately prior to use; additionally, the $n$-nonane and methylbenzene were dried over molecular sieves ( $4 \AA$ ).

TABLE 3. Description of chemical samples, where $x$ is mole fraction, $w$ is mass fraction and $\rho_{\mathrm{e}}$ is electrical resistivity.

\begin{tabular}{llll}
\hline Chemical name & Source & Purity as supplied & $\begin{array}{l}\text { Additional } \\
\text { purification }\end{array}$ \\
\hline Carbon dioxide & BOC & $x \geq 0.99995$ & None \\
Helium & BOC & $x \geq 0.99995$ & None \\
$n$-Nonane & Sigma-Aldrich & $w=0.998$ & Degassed, dried \\
Methylbenzene & Sigma-Aldrich & $w=0.9996$ & Degassed, dried \\
Water & Millipore Direct-Q UV3 & $\begin{array}{l}\rho_{\mathrm{e}}>18 \mathrm{M} \Omega \cdot \mathrm{cm} \text { at } \\
T=298 \mathrm{~K}\end{array}$ & $\begin{array}{l}\text { Degassed under } \\
\text { vacuum }\end{array}$ \\
\hline
\end{tabular}




\subsection{Density}

The density measurements reported in this paper were carried out with a high-pressure vibrating-tube densimeter (VTD, Anton-Paar model DMA HP). The VTD was connected to a master instrument (Anton-Paar, DMA $5000 \mathrm{M}$ ) that displayed the measuring parameters including the temperature, pressure and the period of oscillation. The associated experimental set-up, illustrated in Fig. 1, included the VTD, a high-pressure syringe pump (DH Budenburg) with a maximum working pressure of $70 \mathrm{MPa}$, a sample supply vessel, and a pressure sensor (Keller model $33 \mathrm{X}$ ) with a full-scale range of $70 \mathrm{MPa}$. The VTD was designed to measure the density of liquids and gases at pressures up to $70 \mathrm{MPa}$ and temperatures between (283.15 and 473.15$) \mathrm{K}$. It was fitted with U-shaped hastelloy vibrating-tube, having an internal volume of approximately $2 \mathrm{~mL}$, the temperature of which was controlled by an integrated Peltier thermostat with a stability of $\pm 0.003 \mathrm{~K}$.

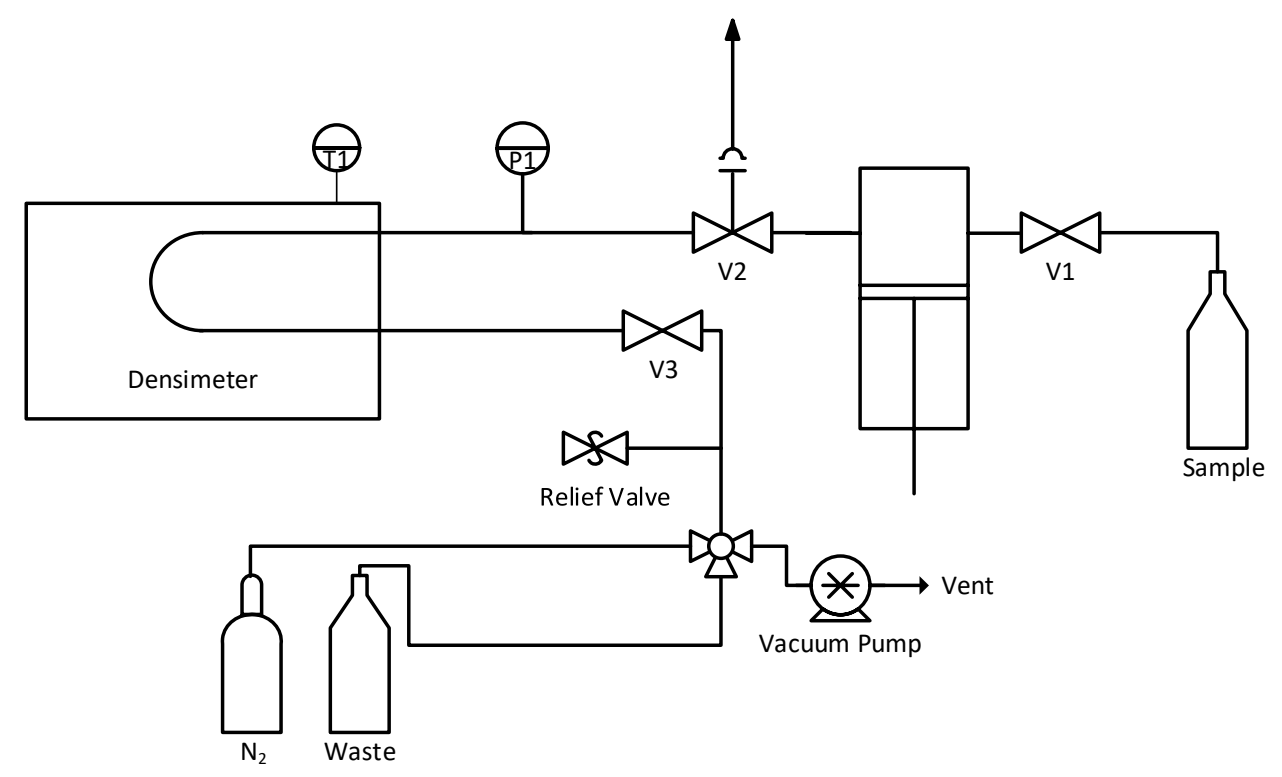

Fig 1. Schematic diagram of the high-pressure densimeter apparatus.

The temperature of the VTD was measured by a $100 \Omega$ platinum resistance thermometer (PRT) integrated within the cellblock, with readout via the DMA 5000. The expanded uncertainty of the temperature, as specified by the manufacturer, is $0.05 \mathrm{~K}$ at $95 \%$ probability. The pressure sensor had both digital and analogue outputs. In this work, the analogue output was used as this permitted integration with the DMA 5000 unit for readout. Operating in this mode, the expanded relative uncertainty was specified by the manufacturer as $0.1 \%$ of full scale, so that the estimated standard uncertainty of pressures was $0.035 \mathrm{MPa}$. The performance of the pressure sensor was verified by comparison with a pneumatic calibrator 
(Fluke-DHI model PPCH-G-70M) having an uncertainty of $0.02 \%$ of reading and observed relative deviations were within $\pm 0.03 \%$ of reading.

The density $\rho$ was obtained from working equation of the VTD:

$$
\rho=A \tau^{2}-B
$$

where $T$ is the period of oscillation and $A$ and $B$ are functions of temperature and pressure obtained by calibration with fluids of known density. In order to establish the values of both $A$ and $B$ without simplifying assumptions, it is necessary to calibrate with two different fluids, ideally with densities that straddle those of the fluid to be measured [35; 36]. In this work, we used helium and water as the calibration fluids in addition to measurements under vacuum. The calibration measurements were carried out at each experimental temperature and over the full working pressure range. It was found that $A$ and $B$ could be correlated accurately as linear functions of pressure along each isotherm. Water and helium were chosen because they have been extensively studied and the available EoS models have low relative standard uncertainties of between $0.0005 \%$ and $0.05 \%$, depending upon the state point $[37 ; 38 ; 39]$. These figures are based on the tolerances specified in [37] and [39], both interpreted as the $k=2$ expanded uncertainty.

Prior to use, the VTD was flushed with nitrogen and dried under vacuum. About $100 \mathrm{~mL}$ of dried and degassed sample was transferred to a glass reservoir with a close-fitting screw cap and a dip tube with $5 \mu \mathrm{m}$ in-line filter through which sample could be withdrawn. The dip tube was connected to the inlet of the high-pressure fluid handling system and the fluid was drawn into the evacuated apparatus. The pressure was raised by means of the syringe pump. The temperatures studied were $283.15 \mathrm{~K}$ and from (298.15 to 473.15$) \mathrm{K}$ in steps of $25 \mathrm{~K}$. On each isotherm, measurements typically started at a pressure of $1 \mathrm{MPa}$, then proceeded from $5 \mathrm{MPa}$ to $65 \mathrm{MPa}$ in steps of $5 \mathrm{MPa}$. The reported results were the averages of two or more measurements made at each state point. Check measurements were also made with decreasing pressure; no hysteresis was observed and the repeatability of the period measurements was within the resolution of $0.01 \mu \mathrm{s}$.

Validation measurements were carried out on methylbenzene at 125 state points distributed over the ranges $283.15 \leq T / \mathrm{K} \leq 473.15$ and $0.1 \leq p \leq 65 \mathrm{MPa}$. The results, given in Table S1 in the Supplementary Material, are compared in Fig. 2 with the reference correlation of McLinden and Splett [40], which is associated with a very small standard uncertainty of 0.0086 $\mathrm{kg} \cdot \mathrm{m}^{-3}$. This comparison is limited to $T \leq 423.15 \mathrm{~K}$ and $p \leq 30 \mathrm{MPa}$ by the region of validity of the equation. It can be seen that our data show small but systematic negative deviations from the reference correlation; these have a root-mean-square value of $0.26 \mathrm{~kg} \cdot \mathrm{m}^{-3}$, or about 
$0.03 \%$ of the median density. We also compared our results over the full experimental range with the equation of state of Lemmon and Span [7], which is associated with a relative uncertainty of $0.05 \%$ or approximately $0.4 \mathrm{~kg} \cdot \mathrm{m}^{-3}$. Table 4 provides a statistical summary of the two comparisons in terms of the mean deviation $\Delta_{\text {bias }}$, the average absolute deviation $\Delta_{\mathrm{AAD}}$, and the maximum absolute deviation $\Delta_{\mathrm{MAD}}$. The deviations fall within the uncertainty of the Lemmon-Span EoS. We note that the correlation of McLinden and Splett agrees with the equation of state of Lemmon and Span to within about $\pm 0.01 \%$ at temperatures between $283 \mathrm{~K}$ and $373 \mathrm{~K}$, and to within $\pm 0.03 \%$ at temperatures up to $423 \mathrm{~K}$.

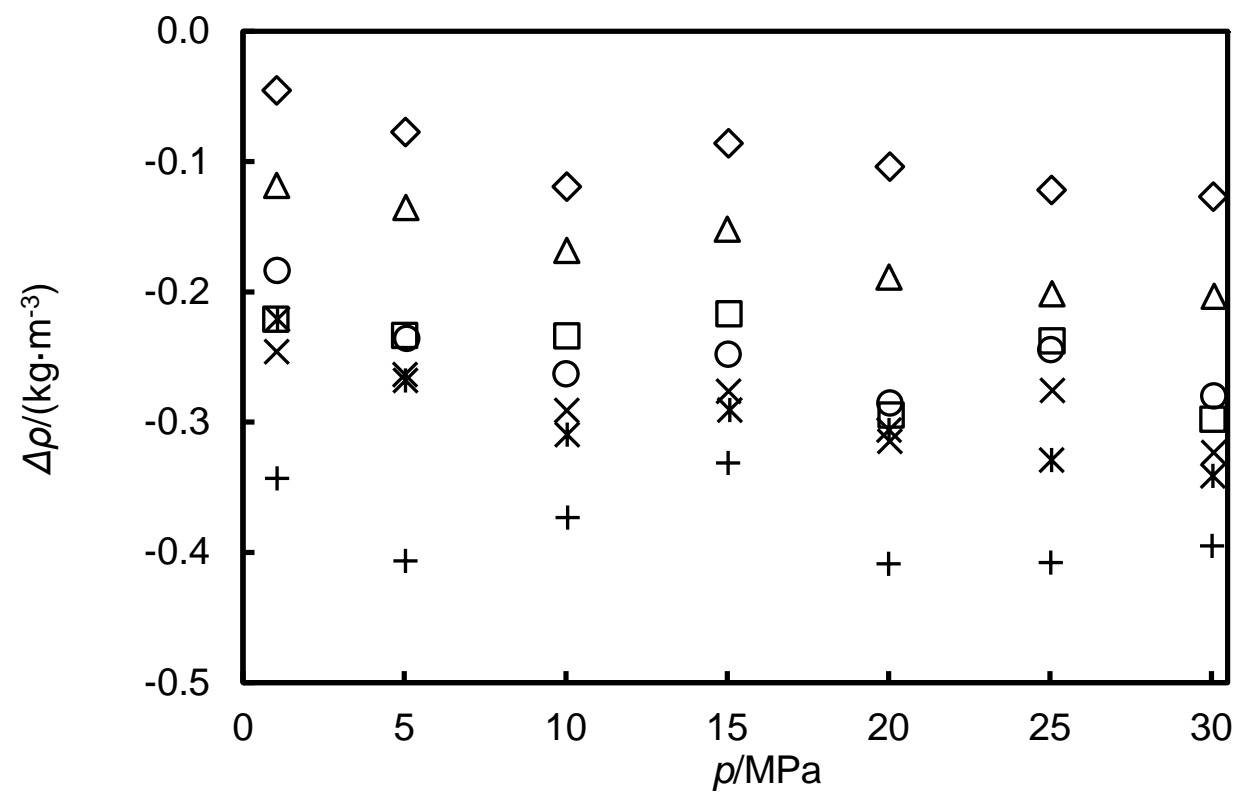

Fig. 2. Deviations $\Delta \rho=\rho-\rho_{\text {calc }}$ between experimental densities of methylbenzene and values $\rho_{\text {calc }}$ calculated from the reference correlation of McLinden and Splett [40] at pressures $p$ and temperatures $T$. Symbols: $\square, T=283.15 \mathrm{~K} ; \diamond, T=298.15 \mathrm{~K} ; \triangle, T=323.15 \mathrm{~K} ; \mathrm{O}$, $T=348.15 \mathrm{~K} ; \times, T=373.15 \mathrm{~K} ; *, T=398.15 \mathrm{~K} ;+, T=423.15 \mathrm{~K}$.

TABLE 4. Comparison of density measurements on methylbenzene with the reference correlation of McLinden and Splett [40] and the equation of state of Lemmon and Span [7]: $\Delta_{\text {bias }}$ is the bias, $\Delta_{\text {RMS }}$ is the root-mean-square deviation, and $\Delta_{\text {MAD }}$ is the maximum absolute deviation. $T_{\min }$ and $T_{\max }$ are the minimum and maximum temperatures, and $p_{\min }$ and $p_{\max }$ are the minimum and maximum pressures, considered in the comparison.

\begin{tabular}{lccccccc}
\hline Model & $T_{\min } / \mathrm{K}$ & $T_{\max } / \mathrm{K}$ & $p_{\min } / \mathrm{MPa}$ & $p_{\max } / \mathrm{MPa}$ & $\Delta_{\text {bias }} /\left(\mathrm{kg} \cdot \mathrm{m}^{-3}\right)$ & $\Delta_{\mathrm{RMS}} /\left(\mathrm{kg} \cdot \mathrm{m}^{-3}\right)$ & $\Delta_{\mathrm{MAD}} /\left(\mathrm{kg} \cdot \mathrm{m}^{-3}\right)$ \\
\hline$[40]$ & 283.15 & 423.15 & 0.1 & 30 & -0.25 & 0.26 & 0.41 \\
{$[7]$} & 283.15 & 473.15 & 0.1 & 65 & -0.20 & 0.25 & 0.44 \\
\hline
\end{tabular}


In order to determine the uncertainty in the measured density, we recast Eq. (1) explicitly in terms of the densities $\rho_{1}$ and $\rho_{2}$ of the calibration fluids and the corresponding periods $\tau_{1}$ and $T_{2}$ measured when these fluids were in the densimeter at the same temperature and pressure as the test fluid:

$$
\rho=\frac{\rho_{1}\left(T^{2}-T_{2}^{2}\right)-\rho_{2}\left(T^{2}-T_{1}^{2}\right)}{\left(T_{1}^{2}-T_{2}^{2}\right)} .
$$

It follows that the square of the standard uncertainty of the test-fluid density is given by

$$
\begin{aligned}
u^{2}(\rho) & =\left[\left(\frac{\partial \rho}{\partial T}\right) u(T)\right]^{2}+\left[\left(\frac{\partial \rho}{\partial T_{1}}\right) u\left(T_{1}\right)\right]^{2}+\left[\left(\frac{\partial \rho}{\partial T_{2}}\right) u\left(T_{2}\right)\right]^{2}+\left[\left(\frac{\partial \rho}{\partial \rho_{1}}\right) u\left(\rho_{1}\right)\right]^{2}+\left[\left(\frac{\partial \rho}{\partial \rho_{2}}\right) u\left(\rho_{2}\right)\right]^{2} \\
& =\left[\frac{2 T\left(\rho_{1}-\rho_{2}\right)}{T_{1}^{2}-T_{2}^{2}} u(T)\right]^{2}+\left[\frac{2 T_{1}\left(\rho_{2}-\rho\right)}{T_{1}^{2}-T_{2}^{2}} u\left(T_{1}\right)\right]^{2}+\left[\frac{2 T_{2}\left(\rho-\rho_{1}\right)}{T_{1}^{2}-T_{2}^{2}} u\left(T_{2}\right)\right]^{2} \\
& +\left[\frac{T^{2}-T_{2}^{2}}{T_{1}^{2}-T_{2}^{2}} u\left(\rho_{1}\right)\right]^{2}+\left[\frac{T_{1}^{2}-T^{2}}{T_{1}^{2}-T_{2}^{2}} u\left(\rho_{2}\right)\right]^{2}+u_{\mathrm{B}}^{2}(\rho)
\end{aligned}
$$

where $u(T), u\left(T_{1}\right), u\left(T_{2}\right)$ are the standard uncertainties of the three period measurements, $u\left(\rho_{1}\right)$ and $u\left(\rho_{2}\right)$ are the standard uncertainties of the reference densities, including the propagated effects of uncertainties in the temperature and pressure during calibration, and $u_{\mathrm{B}}(\rho)$ is the estimated systematic (type-B) uncertainty. The overall combined expanded uncertainty of the density is then calculated from the relation

$$
U_{c}(\rho)=k\left[u^{2}(\rho)+\left\{\left(\frac{\partial \rho}{\partial p}\right)_{T} u(p)\right\}^{2}+\left\{\left(\frac{\partial \rho}{\partial T}\right)_{p} u(T)\right\}\right]^{1 / 2}
$$

where $u(p)$ and $u(T)$ are the uncertainties in pressure and temperature, respectively, and $k$ is the coverage factor. The uncertainty budget for density is detailed in Table 5 for an example case of the median temperature and pressure. The sensitivity factors were estimated from the equation of state of Lemmon and Span [7]. Sample purity was not included here, but calculations using the mixture model in the REFPROP software (version 9.1) [41] suggest that the 0.002 mass fraction impurity would give rise to density changes $<0.02 \mathrm{~kg} \cdot \mathrm{m}^{-3}$. The type-B uncertainty was estimated as the root-mean-square deviation between the validation measurements on methylbenzene and the reference correlation of McLinden and Splett [40]. The overall combined expanded uncertainty $U_{c}(\rho)$ is found to be $\leq 0.6 \mathrm{~kg} \cdot \mathrm{m}^{-3}$ for all state points and, for simplicity, we ascribe that uncertainty to all points. The corresponding overall combined expanded relative uncertainty is $\leq 0.1 \%$. The type-B term is the dominant contribution and may reflect the limitations of a well-calibrated VTD. 
TABLE 5. Uncertainty budget for density at $T=373.15 \mathrm{~K}$ and $p=30 \mathrm{MPa}$, where $T$ is temperature, $p$ is pressure, $\rho$ is density and $t$ is period of oscillation. Subscripts 1 and 2 refer to helium and water, respectively. Sensitivity factors associated with temperature and pressure were estimated from the equation of state of Lemmon and Span [7].

\begin{tabular}{|c|c|c|c|c|}
\hline Quantity & Value & $\begin{array}{l}\text { Standard } \\
\text { uncertainty }\end{array}$ & Sensitivity factor & $\begin{array}{l}\text { Standard } \\
\text { uncertainty }\end{array}$ \\
\hline$\rho_{1}$ & $34.92 \mathrm{~kg} \cdot \mathrm{m}^{-3}$ & $0.17 \mathrm{~kg} \cdot \mathrm{m}^{-3}$ & 0.31 & $0.011 \mathrm{~kg} \cdot \mathrm{m}^{-3}$ \\
\hline$\rho_{2}$ & $971.82 \mathrm{~kg} \cdot \mathrm{m}^{-3}$ & $0.10 \mathrm{~kg} \cdot \mathrm{m}^{-3}$ & 0.70 & $0.019 \mathrm{~kg} \cdot \mathrm{m}^{-3}$ \\
\hline$T_{1}$ & $2479.08 \mu \mathrm{s}$ & $0.01 \mu \mathrm{s}$ & $1.41 \mathrm{~kg} \cdot \mathrm{m}^{-3} \cdot \mu^{-1}$ & $0.014 \mathrm{~kg} \cdot \mathrm{m}^{-3}$ \\
\hline$T_{2}$ & $2673.25 \mu \mathrm{s}$ & $0.01 \mu \mathrm{s}$ & $3.48 \mathrm{~kg} \cdot \mathrm{m}^{-3} \cdot \mu^{-1}$ & $0.035 \mathrm{~kg} \cdot \mathrm{m}^{-3}$ \\
\hline$T$ & $2615.64 \mu \mathrm{s}$ & $0.01 \mu \mathrm{s}$ & $4.90 \mathrm{~kg} \cdot \mathrm{m}^{-3} \cdot \mu^{-1}$ & $0.049 \mathrm{~kg} \cdot \mathrm{m}^{-3}$ \\
\hline$p$ & $30 \mathrm{MPa}$ & $0.035 \mathrm{MPa}$ & $0.90 \mathrm{~kg} \cdot \mathrm{m}^{-3} \cdot \mathrm{MPa}^{-1}$ & $0.031 \mathrm{~kg} \cdot \mathrm{m}^{-3}$ \\
\hline$T$ & $373.15 \mathrm{~K}$ & $0.025 \mathrm{~K}$ & $0.65 \mathrm{~kg} \cdot \mathrm{m}^{-3} \cdot \mathrm{K}^{-1}$ & $0.016 \mathrm{~kg} \cdot \mathrm{m}^{-3}$ \\
\hline \multicolumn{4}{|l|}{ Type B } & $0.26 \mathrm{~kg} \cdot \mathrm{m}^{-3}$ \\
\hline \multicolumn{4}{|c|}{ Expanded uncertainty $(k=2)$} & $\mathrm{kg} \cdot \mathrm{m}^{-3}$ \\
\hline
\end{tabular}

\subsection{Sound speed}

The speed of sound was measured with the dual-path pulse-echo apparatus described in detail previously [42]. The associated fluid-handling system is shown schematically in Fig. 3. The ultrasonic cell had an operating frequency of $5 \mathrm{MHz}$ and was housed in a high-pressure stainless-steel pressure vessel (Sitec-Sieber Engineering AG, model 740.1284) immersed in a thermostatic bath (Fluke model 6020) filled with silicone oil. The thermostatic bath provided a temperature stability and uniformity of $\pm 0.005 \mathrm{~K}$ at temperatures up to $473.15 \mathrm{~K}$. To provide the cooling necessary to achieve temperatures below about $313 \mathrm{~K}$, a heat-exchanger device was immersed in the bath through which ethanol from an external refrigerated circulating bath was passed. The temperature of the pressure vessel was measured with a platinum resistance thermometer (Fluke Model 5615) with standard uncertainty $u_{T}=0.015 \mathrm{~K}$. The pressure was measured with a transducer (Honeywell model TJE/60000) with an estimated standard uncertainty which was the larger of $0.05 \mathrm{MPa}$ and $6 \cdot 10^{-4} \mathrm{p}$. This sensor was calibrated over its full working range in the work of Lin and Trusler [42]. Following this work, the calibration was verified for pressures up to $240 \mathrm{MPa}$ by comparison with a hydraulic pressure balance (DHBudenberg model 580EHX) having a standard relative uncertainty of $0.004 \%$ and the observed deviations were within the uncertainties established by Lin and Trusler. 


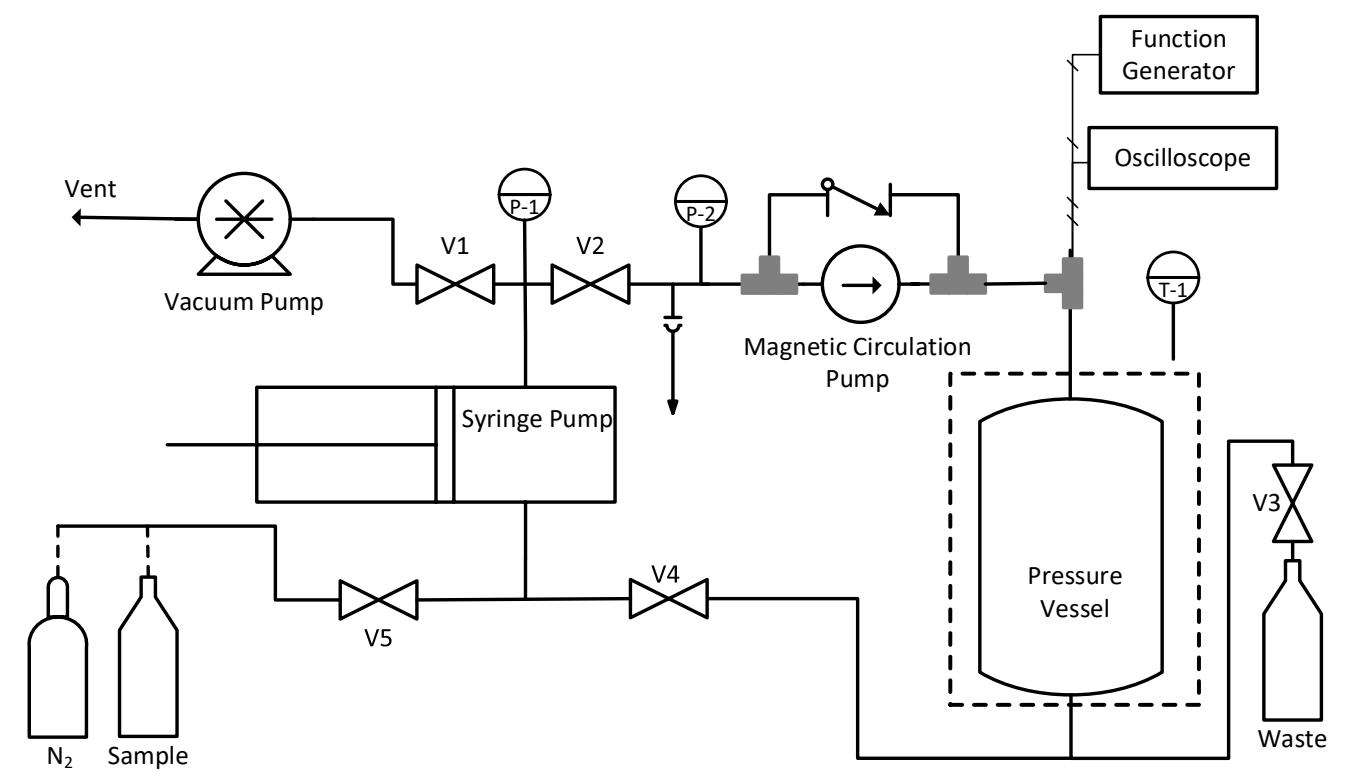

Fig. 3. Schematic diagram of the high-pressure ultrasonic apparatus.

The speed-of-sound $c$ was determined from the relation

$$
c=\frac{2 \Delta L}{\Delta t+T}
$$

where $\Delta L$ is the difference between the long path-length and the short path-length in the ultrasonic cell, and $\Delta t$ is the measured difference between the round-trip times of flight for ultrasonic pulses travelling on the long and the short paths, respectively. Additionally, $T$ is a small correction for diffraction [22; 43] that does not exceed $0.015 \%$ in relative magnitude [42]. The path-length difference was represented on each isotherm by means of the following equation

$$
\Delta L(T, p)=\Delta L_{0}\left[1-\frac{1}{3}\langle\beta\rangle\left(p-p_{0}\right)\right]
$$

in which $\Delta L_{0}$ is the path-length difference at a reference pressure $p_{0}$ and $\langle\beta\rangle$ is the mean value of the isothermal compressibility of the fused-quartz spacer tubes at temperature $T$ over the pressure interval $\left[p_{0}, p\right]$. The value of $\Delta L_{0}$ at each temperature was obtained by calibration with pure water at a reference pressure $p_{0}$ of between ( 1 and 2) MPa, chosen to ensure that the ultrasonic cell was completely filled with liquid. The speed of sound in water was taken from the IAPWS-95 EoS developed by Wagner and Pruss [37] and $\langle\beta\rangle$ were calculated from the correlation developed by Lin and Trusler [42].

Prior to sample injection, the sound-speed apparatus was flushed with nitrogen and dried under vacuum. The dried and degassed sample was transferred to a glass reservoir fitted with a dip tube and $5 \mu \mathrm{m}$ in-line filter; for there it was drawn into the evacuated syringe pump 
through valve $\mathrm{V}-5$ and then injected into the pressure vessel. The measurement along each isotherm started at a pressure of $1 \mathrm{MPa}$ and then proceeded from (30 to 390) MPa in steps of $30 \mathrm{MPa}$; finally, a check measurement was performed at the initial pressure. The measurements were carried out at temperatures of $283.15 \mathrm{~K}$ and from (298.15 to 473.15$) \mathrm{K}$ in steps of $25 \mathrm{~K}$. Two or more measurements were made at each state point and the results were averaged.

To estimate the uncertainty of the speed of sound, Eqs. (5) and (6) were combined, and $\Delta L_{0}$ was eliminated in favour of the time difference $\Delta t_{0}$ measured in the calibration fluid, resulting in the following working equation:

$$
c=c_{0}\left(\frac{\Delta t_{0}+T_{0}}{\Delta t+T}\right)\left[1-\frac{1}{3}\langle\beta\rangle\left(p-p_{0}\right)\right]
$$

Here, $c_{0}$ is the speed of sound in the calibration fluid and $T_{0}$ is the diffraction correction in the calibration experiment. Neglecting (for the uncertainty estimate only) the very small diffraction terms, the square of the standard uncertainty of the speed of sound is then given by

$$
\begin{aligned}
u^{2}(c) & =\left[\left(\frac{\partial c}{\partial c_{0}}\right) u\left(c_{0}\right)\right]^{2}+\left[\left(\frac{\partial c}{\partial \Delta t_{0}}\right) u\left(\Delta t_{0}\right)\right]^{2}+\left[\left(\frac{\partial c}{\partial \Delta t}\right) u(\Delta t)\right]^{2}+\left[\left(\frac{\partial c}{\partial\langle\beta\rangle}\right) u(\langle\beta\rangle)\right]^{2} \\
& =\left[\frac{c}{c_{0}} u\left(c_{0}\right)\right]^{2}+\left[\frac{c}{\Delta t_{0}} u\left(\Delta t_{0}\right)\right]^{2}+\left[\frac{c}{\Delta t} u(\Delta t)\right]^{2}+\left[\frac{-c_{0} \Delta t_{0}\left(p-p_{0}\right)}{3 \Delta t} u(\langle\beta\rangle)\right]^{2}
\end{aligned}
$$

and the overall combined expanded uncertainty $U_{c}(c)$ is given by

$$
U_{c}(c)=k\left[u^{2}(c)+\left\{\left(\frac{\partial c}{\partial p}\right)_{T} u(p)\right\}^{2}+\left\{\left(\frac{\partial c}{\partial T}\right)_{p} u(T)\right\}\right]^{1 / 2}
$$

Input standard uncertainties were taken to be $\Delta t_{0}=\Delta t=0.001 \mu \mathrm{s}$, and $u(\langle\beta\rangle)=0.01 \cdot\langle\beta\rangle$ (based on the analysis present in [42]). In the case of $c_{0}$, the uncertainty was based on the tolerance diagram given in [37] (interpreted as the $k=2$ expanded uncertainty) with additional allowances for the uncertainties of temperature and pressure during calibration. The uncertainty budget for sound speed is exemplified in Table 6 for the case of the median temperature and pressure, at which $c=1829 \mathrm{~m} \cdot \mathrm{s}^{-1}$, leading to an expanded relative uncertainty of $0.11 \%$ with a coverage factor $k=2$. The sensitivity factors were estimated from the equation of state of Lemmon and Span [7]. Again purity was not included but calculation suggest that the effect of likely impurities with mass fraction $w \leq 0.002$ would be $\leq 0.05 \mathrm{~m} \cdot \mathrm{s}^{-1}$. Over the entire region investigated, $u(c) / c$ is nearly constant at $0.05 \%$; however, the terms associated with the derivatives of $c$ with respect to $T$ and, especially, $p$ vary strongly. Over the 
whole surface investigated, we find $U_{c}(c) \leq 2.7 \mathrm{~m} \cdot \mathrm{s}^{-1}$ and that the overall combined expanded relative uncertainty is $\leq 0.32 \%$.

TABLE 6. Uncertainty budget for speed of sound at $T=373.15 \mathrm{~K}$ and $p=210 \mathrm{MPa}$, where $T$ is temperature, $c$ is sound speed, $\Delta t$ is time difference, and $\langle\beta\rangle$ is mean isothermal compressibility of the fused quartz spacer tubes. Subscript 0 refers to water. Sensitivity factors associated with temperature and pressure were estimated from the equation of state of Lemmon and Span [7].

\begin{tabular}{lllll}
\hline Quantity & Value & $\begin{array}{l}\text { Standard } \\
\text { uncertainty }\end{array}$ & Sensitivity factor & $\begin{array}{l}\text { Standard } \\
\text { uncertainty }\end{array}$ \\
\hline$c_{0}$ & $1545.09 \mathrm{~m} \cdot \mathrm{s}^{-1}$ & $0.78 \mathrm{~m} \cdot \mathrm{s}^{-1}$ & 0.924 & $0.915 \mathrm{~m} \cdot \mathrm{s}^{-1}$ \\
$\Delta t_{0}$ & $12.944 \mu \mathrm{s}$ & $0.001 \mu \mathrm{s}$ & $141.3 \mathrm{~m} \cdot \mathrm{s}^{-1} \cdot \mu \mathrm{s}^{-1}$ & $0.141 \mathrm{~m} \cdot \mathrm{s}^{-1}$ \\
$\Delta t$ & $10.913 \mu \mathrm{s}$ & $0.001 \mu \mathrm{s}$ & $167.7 \mathrm{~m} \cdot \mathrm{s}^{-1} \cdot \mu \mathrm{s}^{-1}$ & $0.168 \mathrm{~m} \cdot \mathrm{s}^{-1}$ \\
$\langle\beta\rangle$ & $27 \mathrm{GPa}^{-1}$ & $0.27 \mathrm{GPa}$ & $0.127 \mathrm{~m} \cdot \mathrm{s}^{-1} \cdot \mathrm{GPa}$ & $0.034 \mathrm{~m} \cdot \mathrm{s}^{-1}$ \\
$T$ & $373.15 \mathrm{~K}$ & $0.015 \mathrm{~K}$ & $1.89 \mathrm{~m} \cdot \mathrm{s}^{-1} \cdot \mathrm{K}^{-1}$ & $0.028 \mathrm{~m} \cdot \mathrm{s}^{-1}$ \\
$p$ & 210.00 & $0.13 \mathrm{MPa}$ & $2.79 \mathrm{~m} \cdot \mathrm{s}^{-1} \cdot \mathrm{MPa} \mathrm{Ma}^{-1}$ & $0.351 \mathrm{~m} \cdot \mathrm{s}^{-1}$ \\
\hline \multicolumn{3}{c}{$2.0 \mathrm{~m} \cdot \mathrm{s}^{-1}$} \\
\hline
\end{tabular}

\section{Experimental Results and Correlations}

\subsection{Density}

The density of $n$-nonane was measured along nine isotherms at $T=(283.15,298.15,323.15$, $348.15,373.15,398.15,423.15,448.15$ and 473.15$) \mathrm{K}$ and at pressures between (0.1 and 65) $\mathrm{MPa}$, except on the highest isotherm where the measurements were confined to $p \geq 5 \mathrm{MPa}$. Check measurements were included in which the density at $p \approx 5 \mathrm{MPa}$ was re-measured after completing all higher pressures on the same isotherm; in all cases, the differences were smaller in magnitude than $0.05 \mathrm{~kg} \cdot \mathrm{m}^{-3}$. The experimental results are given in Table 7 and plotted in Fig. 4(a).

Table 7. Density $\rho$ for $n$-nonane at temperatures $T$ and pressure $p .^{\text {a }}$

\begin{tabular}{|c|c|c|c|c|c|}
\hline$p / \mathrm{MPa}$ & $\rho /\left(\mathrm{kg} \cdot \mathrm{m}^{-3}\right)$ & $p / \mathrm{MPa}$ & $\rho /\left(\mathrm{kg} \cdot \mathrm{m}^{-3}\right)$ & $\mathrm{p} / \mathrm{MPa}$ & $\rho /\left(\mathrm{kg} \cdot \mathrm{m}^{-3}\right)$ \\
\hline \multicolumn{6}{|c|}{$T=283.15 \mathrm{~K}$} \\
\hline 1.01 & 726.22 & 25.02 & 742.54 & 50.01 & 756.62 \\
\hline 5.04 & 729.17 & 30.04 & 745.52 & 55.02 & 759.19 \\
\hline 10.02 & 732.75 & 35.05 & 748.45 & 60.03 & 761.66 \\
\hline 15.02 & 736.14 & 40.01 & 751.23 & 65.01 & 764.08 \\
\hline 20.04 & 739.41 & 45.05 & 753.99 & 5.02 & 729.22 \\
\hline \multicolumn{6}{|c|}{$T=298.15 \mathrm{~K}$} \\
\hline 1.02 & 714.83 & 25.04 & 732.54 & 50.01 & 747.56 \\
\hline 5.01 & 718.08 & 30.01 & 735.76 & 55.04 & 750.31 \\
\hline 10.03 & 721.94 & 35.05 & 738.87 & 60.02 & 752.92 \\
\hline 15.06 & 725.70 & 40.01 & 741.88 & 65.01 & 755.48 \\
\hline
\end{tabular}




\begin{tabular}{|c|c|c|c|c|c|}
\hline $\mathrm{p} / \mathrm{MPa}$ & $\rho /\left(\mathrm{kg} \cdot \mathrm{m}^{-3}\right)$ & $p / \mathrm{MPa}$ & $\rho /\left(\mathrm{kg} \cdot \mathrm{m}^{-3}\right)$ & $p / \mathrm{MPa}$ & $\rho /\left(\mathrm{kg} \cdot \mathrm{m}^{-3}\right)$ \\
\hline 20.04 & 729.17 & 45.06 & 744.79 & 5.04 & 718.12 \\
\hline \multicolumn{6}{|c|}{$T=323.15 \mathrm{~K}$} \\
\hline 1.03 & 695.27 & 25.02 & 715.63 & 50.03 & 732.36 \\
\hline 5.03 & 699.04 & 30.02 & 719.22 & 55.05 & 735.35 \\
\hline 10.05 & 703.57 & 35.08 & 722.75 & 60.01 & 738.19 \\
\hline 15.02 & 707.81 & 40.05 & 726.09 & 65.01 & 741.02 \\
\hline 20.05 & 711.84 & 45.06 & 729.27 & 5.06 & 699.09 \\
\hline \multicolumn{6}{|c|}{$T=348.15 \mathrm{~K}$} \\
\hline 1.01 & 675.18 & 25.05 & 698.79 & 50.04 & 717.37 \\
\hline 5.04 & 679.76 & 30.03 & 702.84 & 55.05 & 720.67 \\
\hline 10.03 & 685.00 & 35.05 & 706.74 & 60.00 & 723.79 \\
\hline 15.02 & 689.84 & 40.02 & 710.40 & 65.04 & 726.84 \\
\hline 20.04 & 694.44 & 45.07 & 714.01 & 5.03 & 679.76 \\
\hline \multicolumn{6}{|c|}{$T=373.15 \mathrm{~K}$} \\
\hline 1.01 & 654.52 & 25.06 & 681.94 & 50.07 & 702.65 \\
\hline 5.05 & 659.99 & 30.04 & 686.51 & 55.06 & 706.23 \\
\hline 10.03 & 666.13 & 35.06 & 690.83 & 60.04 & 709.66 \\
\hline 15.07 & 671.78 & 40.02 & 694.95 & 65.03 & 712.95 \\
\hline 20.03 & 677.04 & 45.07 & 698.87 & 5.05 & 659.99 \\
\hline \multicolumn{6}{|c|}{$T=398.15 \mathrm{~K}$} \\
\hline 1.02 & 633.06 & 25.03 & 665.05 & 50.02 & 688.07 \\
\hline 5.02 & 639.53 & 30.05 & 670.22 & 55.04 & 692.01 \\
\hline 10.05 & 646.89 & 35.05 & 675.10 & 60.03 & 695.72 \\
\hline 15.07 & 653.53 & 40.02 & 679.63 & 65.04 & 699.37 \\
\hline 20.02 & 659.49 & 45.07 & 683.97 & 5.03 & 639.58 \\
\hline \multicolumn{6}{|c|}{$T=423.15 \mathrm{~K}$} \\
\hline 1.02 & 610.45 & 25.04 & 648.10 & 50.04 & 673.70 \\
\hline 5.03 & 618.42 & 30.02 & 653.92 & 55.02 & 677.97 \\
\hline 10.03 & 627.15 & 35.05 & 659.35 & 60.03 & 682.03 \\
\hline 15.03 & 634.86 & 40.02 & 664.40 & 65.04 & 685.95 \\
\hline 20.04 & 641.80 & 45.02 & 669.15 & 5.03 & 618.47 \\
\hline \multicolumn{6}{|c|}{$T=448.15 \mathrm{~K}$} \\
\hline 1.00 & 586.37 & 25.04 & 631.27 & 50.02 & 659.58 \\
\hline 5.02 & 596.41 & 30.03 & 637.78 & 55.04 & 664.24 \\
\hline 10.04 & 607.05 & 35.03 & 643.80 & 60.03 & 668.62 \\
\hline 15.01 & 616.06 & 40.01 & 649.38 & 65.02 & 672.85 \\
\hline 20.01 & 624.00 & 45.05 & 654.67 & 5.02 & 596.46 \\
\hline \multicolumn{6}{|c|}{$T=473.15 \mathrm{~K}$} \\
\hline & & 25.05 & 614.28 & 50.04 & 645.60 \\
\hline 5.01 & 573.14 & 30.02 & 621.60 & 55.02 & 650.62 \\
\hline 10.04 & 586.15 & 35.03 & 628.30 & 60.01 & 655.39 \\
\hline 15.04 & 596.86 & 40.05 & 634.51 & 65.01 & 659.92 \\
\hline 20.02 & 606.05 & 45.05 & 640.25 & 5.01 & 573.14 \\
\hline
\end{tabular}

a Expanded uncertainties are $U(T)=0.05 \mathrm{~K}, U(p)=0.07 \mathrm{MPa}$ and $U_{\mathrm{c}}(\rho)=0.6 \mathrm{~kg} \cdot \mathrm{m}^{-3}$ with a coverage factor $k=2$.

The density data have been correlated using the modified Tait equation which is recognised as an effective empirical model for compressed liquid density [44]. In terms of density, the modified Tait equation is 


$$
\rho=\rho_{0}\left[1-C \log _{10}\left(\frac{p+B}{p_{0}+B}\right)\right]^{-1},
$$

where $\rho_{0}$ is the density at a reference pressure $p_{0}=0.1 \mathrm{MPa}, C$ is a temperature-independent constant and $B$ is a temperature-dependent parameter. In this work, $\rho_{0}$ and $B$ were represented as functions of temperature by means of the following functions:

$$
\begin{gathered}
\rho_{0} /\left(\mathrm{kg} \cdot \mathrm{m}^{-3}\right)=a_{0}+a_{1} I^{1 / 3}+a_{2} T^{2}+a_{4} T^{4}, \\
B / \mathrm{MPa}=b_{0}+b_{1} T^{2}+b_{2} T^{6},
\end{gathered}
$$

where $T=\left(T_{\mathrm{c}}-T\right) / T_{\mathrm{c}}$ and $T_{\mathrm{c}}$ is the critical temperature of $n$-nonane taken from reference [7]. In this analysis, $\rho_{0}$ refers to hypothetical liquid states at temperatures above approximately $423 \mathrm{~K}$ where the vapour pressure of $n$-nonane exceeds $p_{0}$. The 8 parameters $\left(a_{i}, b_{i}\right.$ and $\left.C\right)$ were adjusted in a non-linear least-squares regression and the optimal values are given in Table 8. Isotherms calculated from the model are plotted in Fig. 4(a) and the relative deviations of the experimental data from the model are shown in Fig. 4(b).

TABLE 8. Parameters in the modified Tait correlation, equations (10 to 12).

\begin{tabular}{ccccccccc}
\hline$T_{\mathrm{c}} / \mathrm{K}$ & $a_{0}$ & $a_{1}$ & $a_{2}$ & $a_{3}$ & $b_{0}$ & $b_{1}$ & $b_{2}$ & $c$ \\
\hline 594.55 & 175.801 & 638.481 & 133.914 & -21.801 & 1.693 & 272.729 & 315.654 & 0.2011 \\
\hline
\end{tabular}

The goodness of fit may be conveniently assessed in terms of the absolute average relative deviation, $\Delta_{\mathrm{AARD}}(\rho)$, and the maximum absolute relative deviation, $\Delta_{\mathrm{MARD}}(\rho)$. These metrics are defined for a property $X$ as follows:

$$
\begin{aligned}
& \Delta_{\mathrm{AARD}}(X)=\frac{1}{N} \sum_{i=1}^{N}\left(\frac{\left|X_{i}-X_{i, \mathrm{fit}}\right|}{X_{i}}\right) \\
& \Delta_{\mathrm{MARD}}(X)=\operatorname{Max}\left(\frac{\left|X_{i}-X_{i, \mathrm{fit}}\right|}{X_{i}}\right),
\end{aligned}
$$

where $N$ is the number of experimental data points $X_{i}$, and $X_{i, \text { fit }}$ are the corresponding values calculated from a fitted equation. As shown in Fig. 4, the modified Tait equation was found to represent the experimental data exceptionally well, and we found $\Delta_{\operatorname{AARD}}(\rho)=0.006 \%$ and $\Delta_{\text {MARD }}(\rho)=0.021 \%$; in terms of density, deviations were all within $\pm 0.15 \mathrm{~kg} \cdot \mathrm{m}^{-3}$, which is well within the expanded uncertainty. Given the wide ranges of temperature and pressure studied, the large number of data points, and the smoothness of the experimental data, the inclusion 
of 8 parameters in the correlation appears to be justified. The correlation is recommended for interpolation but not for extrapolation.

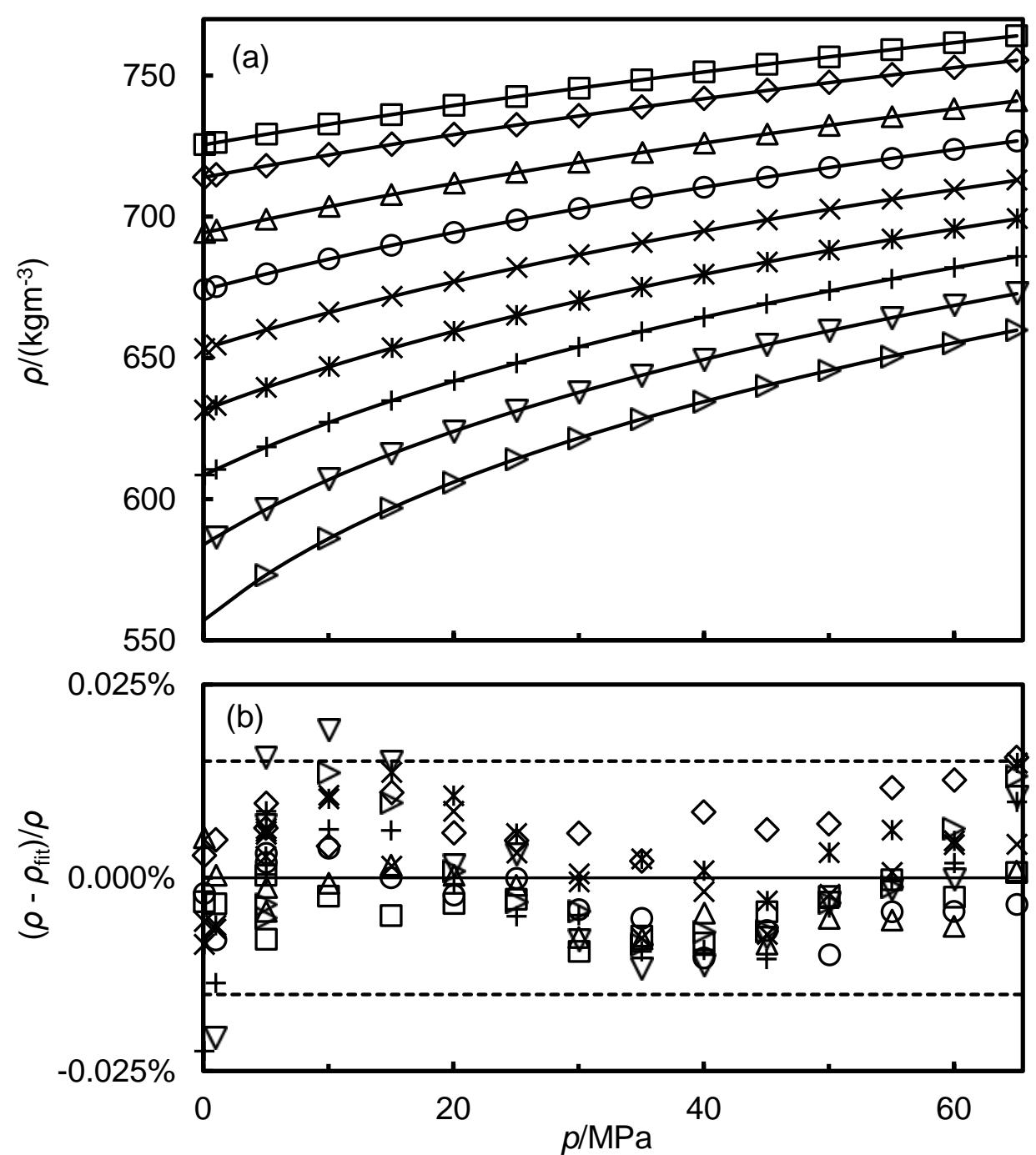

Fig. 4. (a) Experimental density $\rho$ of $n$-nonane as a function of pressure $p$; (b) relative deviations $\left(\rho-\rho_{\mathrm{Fit}}\right) / \rho$ of experimental densities $\rho$ of $n$-nonane from the values $\rho_{\text {fit }}$ obtained from the Tait equation. Symbols: $\square, T=283.15 \mathrm{~K} ; \diamond, T=298.15 \mathrm{~K} ; \triangle, T=323.15 \mathrm{~K} ; O$, $T=348.15 \mathrm{~K} ; \times, T=373.15 \mathrm{~K} ; *, T=398.15 \mathrm{~K} ; \quad+, T=423.15 \mathrm{~K} ; \nabla, T=448.15 \mathrm{~K} ; \quad \square$, $T=473.15 \mathrm{~K}$. Lines: —_ Eq. (10), with parameters from Table 8; - - -, $95 \%$ confidence interval of the correlation.

\subsection{Speed of sound}

The speed of sound was measured along nine isotherms at $T=(283.15,298.15,323.15$, $348.15,373.15,398.15,423.15,448.15$ and 473.15$) \mathrm{K}$ and at pressures up to $390 \mathrm{MPa}$. Check measurements were included in which the initial low-pressure state was re-measured after completing the other pressures on an isotherm. In all cases, the deviations between the initial measurement and the final check measurement at the same temperature and pressure were 
smaller in magnitude than $0.2 \mathrm{~m} \cdot \mathrm{s}^{-1}$. The results are given in Table 9 and plotted in Fig. 5(a). In the region studied, the speed-of-sound ranges from approximately (570 to 2380 ) $\mathrm{m} \cdot \mathrm{s}^{-1}$.

The experimental sound speed data were correlated by means of the following implicit empirical equation:

$$
\left(p-p_{0}\right) / \mathrm{MPa}=\sum_{i=1}^{3} \sum_{j=0}^{3} c_{i j}\left[\left(c-c_{0}\right) /\left(\mathrm{m} \cdot \mathrm{s}^{-1}\right)\right]^{i} \tau^{j}
$$

where $p_{0}=0.1 \mathrm{MPa}, T=\left(T_{\mathrm{c}}-T\right) / T_{\mathrm{c}}$ and $c_{0}=c\left(T, p_{0}\right)$ was correlated as follows:

$$
c_{0} /\left(\mathrm{m} \cdot \mathrm{s}^{-1}\right)=\sum_{i=0}^{3} d_{i} T^{i}
$$

TABLE 9. Speed of sound $c$ in $n$-nonane at temperature $T$ and pressure $p$, and combined

\begin{tabular}{|c|c|c|c|c|c|}
\hline$p / \mathrm{MPa}$ & $c /\left(m \cdot s^{-1}\right)$ & $U_{c}(c) /\left(m \cdot s^{-1}\right)$ & $p / \mathrm{MPa}$ & $c /\left(m \cdot s^{-1}\right)$ & $U_{c}(c) /\left(m \cdot s^{-1}\right)$ \\
\hline \multicolumn{6}{|c|}{$T=283.13 \mathrm{~K}$} \\
\hline 1.09 & 1273.92 & 1.41 & 210.07 & 2017.34 & 2.12 \\
\hline 30.10 & 1432.40 & 1.52 & 240.00 & 2086.26 & 2.21 \\
\hline 59.97 & 1563.71 & 1.62 & 270.32 & 2151.85 & 2.28 \\
\hline 90.37 & 1676.83 & 1.72 & 300.01 & 2212.51 & 2.36 \\
\hline 120.25 & 1774.65 & 1.84 & 330.30 & 2271.03 & 2.43 \\
\hline 149.99 & 1862.22 & 1.94 & 360.22 & 2325.98 & 2.49 \\
\hline 180.13 & 1943.20 & 2.04 & 389.88 & 2378.05 & 2.56 \\
\hline & & & 1.09 & 1273.92 & 1.41 \\
\hline \multicolumn{6}{|c|}{$T=298.15 \mathrm{~K}$} \\
\hline 1.04 & 1212.57 & 1.38 & 210.41 & 1983.68 & 2.10 \\
\hline 30.04 & 1380.17 & 1.47 & 240.02 & 2053.12 & 2.18 \\
\hline 60.13 & 1517.95 & 1.58 & 270.23 & 2119.62 & 2.26 \\
\hline 90.11 & 1633.44 & 1.68 & 300.10 & 2181.40 & 2.33 \\
\hline 120.20 & 1734.73 & 1.80 & 330.26 & 2240.53 & 2.40 \\
\hline 150.12 & 1824.93 & 1.91 & 360.03 & 2295.97 & 2.47 \\
\hline 180.37 & 1907.90 & 2.01 & 390.03 & 2349.18 & 2.53 \\
\hline & & & 1.04 & 1212.57 & 1.38 \\
\hline \multicolumn{6}{|c|}{$T=323.14 \mathrm{~K}$} \\
\hline 1.02 & 1112.35 & 1.33 & 210.41 & 1929.20 & 2.05 \\
\hline 30.05 & 1297.76 & 1.41 & 239.98 & 2000.37 & 2.13 \\
\hline 60.21 & 1445.17 & 1.51 & 270.33 & 2068.72 & 2.21 \\
\hline 90.15 & 1566.72 & 1.62 & 300.07 & 2131.55 & 2.29 \\
\hline 120.24 & 1672.31 & 1.75 & 330.05 & 2191.32 & 2.36 \\
\hline 150.13 & 1765.55 & 1.85 & 360.04 & 2247.86 & 2.43 \\
\hline 179.98 & 1850.08 & 1.95 & 390.06 & 2301.72 & 2.49 \\
\hline & & & 1.02 & 1112.35 & 1.33 \\
\hline \multicolumn{6}{|c|}{$T=348.14 \mathrm{~K}$} \\
\hline 1.06 & 1018.06 & 1.30 & 210.23 & 1877.75 & 2.00 \\
\hline 30.15 & 1220.84 & 1.35 & 240.36 & 1951.75 & 2.09 \\
\hline
\end{tabular}
expanded uncertainty $U_{c}(c)$ of the speed of sound with a coverage factor of 2 . $^{a}$ 


\begin{tabular}{|c|c|c|c|c|c|}
\hline$p / \mathrm{MPa}$ & $c /\left(m \cdot s^{-1}\right)$ & $U_{c}(c) /\left(m \cdot s^{-1}\right)$ & $p / \mathrm{MPa}$ & $c /\left(m \cdot s^{-1}\right)$ & $U_{\mathrm{c}}(\mathrm{c}) /\left(\mathrm{m} \cdot \mathrm{s}^{-1}\right)$ \\
\hline 59.87 & 1375.25 & 1.45 & 269.99 & 2019.19 & 2.17 \\
\hline 90.06 & 1503.76 & 1.57 & 300.42 & 2084.34 & 2.25 \\
\hline 120.21 & 1613.80 & 1.69 & 330.17 & 2144.47 & 2.32 \\
\hline 150.18 & 1710.39 & 1.81 & 360.17 & 2202.19 & 2.39 \\
\hline 180.31 & 1798.07 & 1.91 & 390.07 & 2256.51 & 2.45 \\
\hline \multicolumn{6}{|c|}{$T=373.14 \mathrm{~K}$} \\
\hline 1.02 & 924.94 & 1.29 & 210.01 & 1829.26 & 1.96 \\
\hline 30.12 & 1147.94 & 1.30 & 240.37 & 1905.46 & 2.05 \\
\hline 60.04 & 1312.40 & 1.40 & 270.13 & 1974.68 & 2.13 \\
\hline 90.42 & 1446.98 & 1.51 & 300.13 & 2040.35 & 2.21 \\
\hline 120.19 & 1559.47 & 1.65 & 330.24 & 2102.26 & 2.28 \\
\hline 150.20 & 1659.02 & 1.76 & 360.09 & 2160.06 & 2.35 \\
\hline 180.54 & 1749.05 & 1.87 & 389.86 & 2212.89 & 2.41 \\
\hline & & & 1.02 & 924.94 & 1.29 \\
\hline \multicolumn{6}{|c|}{$T=398.14 \mathrm{~K}$} \\
\hline 1.10 & 835.44 & 1.33 & 210.02 & 1784.32 & 1.92 \\
\hline 30.14 & 1079.80 & 1.26 & 240.15 & 1861.34 & 2.01 \\
\hline 60.24 & 1254.06 & 1.35 & 270.21 & 1933.08 & 2.10 \\
\hline 90.22 & 1391.91 & 1.47 & 299.86 & 1999.64 & 2.17 \\
\hline 120.20 & 1508.49 & 1.60 & 330.01 & 2063.66 & 2.25 \\
\hline 150.15 & 1610.25 & 1.72 & 360.01 & 2123.99 & 2.32 \\
\hline 180.17 & 1701.67 & 1.83 & 389.95 & 2182.15 & 2.39 \\
\hline & & & 1.10 & 835.46 & 1.33 \\
\hline \multicolumn{6}{|c|}{$T=423.14 \mathrm{~K}$} \\
\hline 1.11 & 747.67 & 1.41 & 210.18 & 1744.05 & 1.89 \\
\hline 29.69 & 1013.62 & 1.23 & 239.99 & 1822.32 & 1.98 \\
\hline 60.21 & 1199.10 & 1.31 & 270.12 & 1895.69 & 2.06 \\
\hline 90.05 & 1341.02 & 1.42 & 299.98 & 1964.43 & 2.14 \\
\hline 120.57 & 1462.66 & 1.56 & 330.04 & 2029.42 & 2.22 \\
\hline 150.11 & 1565.38 & 1.68 & 360.02 & 2089.73 & 2.29 \\
\hline 180.16 & 1658.79 & 1.79 & 389.72 & 2146.05 & 2.36 \\
\hline & & & 1.12 & 747.72 & 1.41 \\
\hline \multicolumn{6}{|c|}{$T=448.15 \mathrm{~K}$} \\
\hline 1.04 & 660.90 & 1.57 & 209.90 & 1704.89 & 1.85 \\
\hline 30.44 & 960.29 & 1.21 & 239.92 & 1784.34 & 1.95 \\
\hline 60.49 & 1150.48 & 1.27 & 270.08 & 1858.36 & 2.03 \\
\hline 90.22 & 1295.98 & 1.38 & 299.83 & 1929.76 & 2.12 \\
\hline 120.32 & 1418.80 & 1.52 & 330.05 & 1995.92 & 2.19 \\
\hline 150.28 & 1525.01 & 1.65 & 359.98 & 2059.17 & 2.27 \\
\hline 180.15 & 1619.38 & 1.76 & 390.31 & 2115.06 & 2.33 \\
\hline \multicolumn{6}{|c|}{$T=473.15 \mathrm{~K}$} \\
\hline 1.01 & 574.55 & 1.83 & 210.23 & 1675.78 & 1.83 \\
\hline 30.43 & 906.04 & 1.19 & 240.07 & 1755.44 & 1.92 \\
\hline 60.39 & 1104.62 & 1.24 & 270.13 & 1830.79 & 2.01 \\
\hline 90.20 & 1255.37 & 1.35 & 300.08 & 1901.86 & 2.09 \\
\hline 120.10 & 1381.03 & 1.49 & 330.03 & 1964.77 & 2.17 \\
\hline 150.23 & 1490.59 & 1.62 & 360.07 & 2026.31 & 2.24 \\
\hline 180.26 & 1587.61 & 1.73 & 390.06 & 2083.97 & 2.31 \\
\hline
\end{tabular}

${ }^{a}$ Expanded uncertainties are $U(T)=0.03 \mathrm{~K}$ and $U(p)=\operatorname{Max}(0.1 \mathrm{MPa}, 0.0012 \cdot p)$ at a coverage factor of 2. 
TABLE 10. Coefficients in the sound-speed correlation, equations (15) and (16). To avoid rounding errors, values are given to more significant figures than justified by the precision of the data.

\begin{tabular}{cccc}
\hline$c_{10}$ & $c_{11}$ & $c_{12}$ & $c_{13}$ \\
$7.257857 \cdot 10^{-2}$ & $-4.009566 \cdot 10^{-1}$ & $1.804538 \cdot 10^{0}$ & $-1.391835 \cdot 10^{0}$ \\
\hline$c_{20}$ & $c_{21}$ & $c_{22}$ & $c_{23}$ \\
$-1.643746 \cdot 10^{-4}$ & $2.273602 \cdot 10^{-3}$ & $-6.295787 \cdot 10^{-3}$ & $5.863198 \cdot 10^{-3}$ \\
\hline$c_{30}$ & $c_{31}$ & $c_{32}$ & $c_{33}$ \\
$1.633157 \cdot 10^{-7}$ & $-1.293797 \cdot 10^{-6}$ & $3.847817 \cdot 10^{-6}$ & $-3.565298 \cdot 10^{-6}$ \\
\hline$d_{0}$ & $d_{1}$ & $d_{2}$ & $d_{3}$ \\
$1.276293 \cdot 10^{2}$ & $2.185110 \cdot 10^{3}$ & $-6.009274 \cdot 10^{3}$ & $1.116485 \cdot 10^{3}$ \\
\hline
\end{tabular}
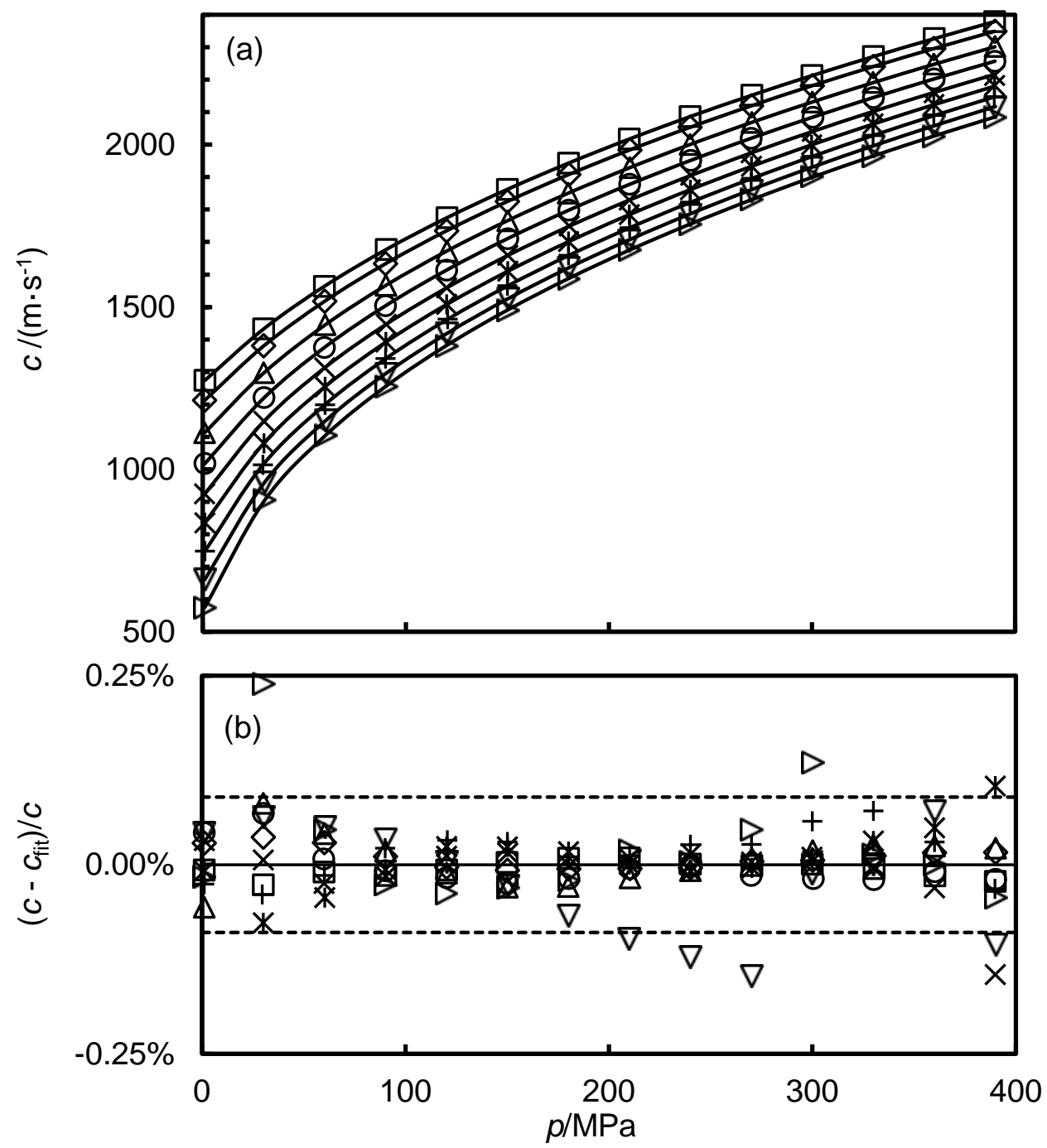

Fig. 5. (a) Experimental speeds of sound of $n$-nonane as a function of pressure $p$; (b) relative deviations $\left(c-c_{\mathrm{fit}}\right) / c$ of the experimental speed of sound $c$ in $n$-nonane from the values $c_{\mathrm{fit}}$ calculated from the surface fit. Symbols: $\square, T=283.15 \mathrm{~K} ; \diamond, T=298.15 \mathrm{~K} ; \triangle, T=323.15 \mathrm{~K}$; $\mathrm{O}, T=348.15 \mathrm{~K} ; \times, T=373.15 \mathrm{~K} ; *, T=398.15 \mathrm{~K} ;+, T=423.15 \mathrm{~K} ; \nabla, T=448.15 \mathrm{~K} ; \nabla$, $T=473.15 \mathrm{~K}$. Lines: ——, Eq. (15), with parameters from Table 10; - - - , $95 \%$ confidence interval of the correlation. 
The parameters in equations (15) and (16) are given in Table 10. Isotherms calculated from the model are plotted in Fig. 5(a) and relative deviations of the experimental data from this surface are shown in Fig. 5(b). The goodness of the fit is summarised by $\Delta_{\text {AARD }}(c)=0.03 \%$ and $\Delta_{\text {MARD }}(c)=0.24 \%$. Figure $5(b)$ shows that the vast majority of points are represented by the surface fit to within $\pm 0.1 \%$.

\section{Derived Thermodynamic Properties}

Having determined the speed of sound over wide ranges of temperature and pressure, we proceeded to calculate a full set of derived properties in the same domain by means of thermodynamic integration, based on the following exact relations:

$$
\begin{gathered}
c^{2}=\left[\left(\frac{\partial \rho}{\partial p}\right)_{T}-\frac{T}{\rho^{2} c_{p}}\left(\frac{\partial \rho}{\partial T}\right)_{p}^{2}\right]^{-1} \\
\left(\frac{\partial c_{p}}{\partial p}\right)=-\frac{T}{\rho^{3}}\left[2\left(\frac{\partial \rho}{\partial T}\right)_{p}^{2}-\rho\left(\frac{\partial^{2} \rho}{\partial T^{2}}\right)_{p}\right]
\end{gathered}
$$

The solution of these coupled partial differential equations is subject to initial values of density and isobaric specific heat capacity along an initial isobar, chosen in this work to be $p_{0}=0.1 \mathrm{MPa}$. The method was first used by Davis and Gordon [45] for mercury and improved by Sun et al. [46]. The algorithm used [47] and the uncertainty of the method have also been studied in detail in a recent paper [48].

Initial values of density were determined from equation (11). However, as the lowest pressure at which the density was measured at $T=473.15 \mathrm{~K}$ was $5 \mathrm{MPa}$, this correlation may represent a poor estimate of the hypothetical liquid density extrapolated to $p=0.1 \mathrm{MPa}$. Therefore, although the integration was carried out at temperatures up to $473.15 \mathrm{~K}$, results at temperatures above $448.15 \mathrm{~K}$ were discarded.

The isobaric specific heat capacity $c_{p}$ was not measured in this work and, instead, a correlation was developed based on the available literature. The nine data sources detailed in Table 2 were considered together with values of $c_{p}$ extrapolated to $p=p_{0}$ from the data of Banipal et al. [13] and Kuznetsov et al. [18]. The data of Banipal et al. [13] relate to pressures up to $10 \mathrm{MPa}$ and temperatures of (318.15 to 373.15$) \mathrm{K}$; these were adequately represented along isotherms as linear functions of pressure. In the case of the Kuznetsov et al. [18] data, the pressure range extends up to $60 \mathrm{MPa}$; quadratic polynomials were fitted on each isotherm up to a greatest temperature of $494 \mathrm{~K}$. The combined data set contained results from 11 authors 
and extended in temperature from $274 \mathrm{~K}$ to $494 \mathrm{~K}$. The data were mostly found to be consistent within the claimed uncertainties, except in the region between $363.15 \mathrm{~K}$ and $373.15 \mathrm{~K}$, where three points of Banipal et al. [13] are significantly higher than a smooth curve through the other available data. Therefore those three points were assigned zero weight in the analysis and the remaining data were found to be represented adequately by a simple quadratic function of temperature as follows:

$$
c_{p} /\left(\mathrm{J} \cdot \mathrm{K}^{-1} \cdot \mathrm{kg}^{-1}\right)=\sum_{i=0}^{2} e_{i}\left(T / T_{0}\right)^{i}
$$

where $T_{0}=273.15 \mathrm{~K}$.

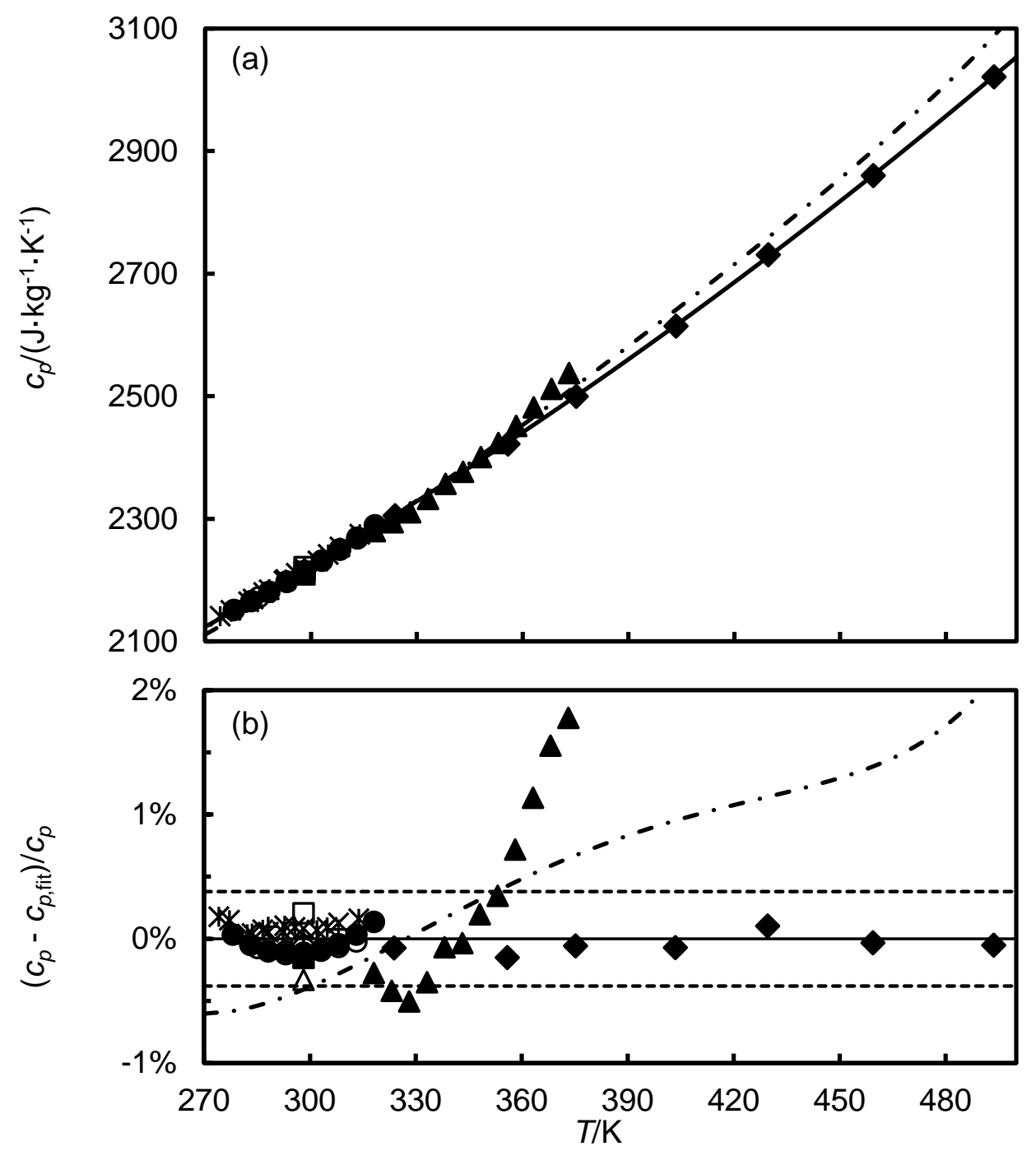

Fig. 6. (a) Isobaric specific heat capacities of $n$-nonane plotted as a function of temperature $T$; (b) relative deviations $\left(c_{p}-c_{p, \text { fit }}\right) / c_{p}$ of the literature heat capacities from the values $c_{p \text {,fit }}$ calculated from Eq. (20). Symbols: $\square$, [26]; $\diamond,[27] ; \triangle$, [28]; O, [29]; $\times$, [30]; *, [31]; +, [32]; 口, [33]; $\bullet$ [18]; $\boldsymbol{\Lambda}$, [13]; ○, [34]. Curves: —, Eq. (19) with parameters from Table 11; $-\cdot-\cdot--$, equation of state of Lemmon and Span [7]; - - - , $95 \%$ confidence interval of the correlation. 
The parameters of Eq. (19) are given in Table 11 and Fig. 6 compares the data with the correlation and also with values obtained from the equation of state of Lemmon and Span [7]. We observe good agreement with between the data and Eq. (19) with $\Delta_{\mathrm{AARD}}\left(c_{p}\right)=0.13 \%$ and $\Delta_{\text {MARD }}\left(c_{p}\right)=0.72 \%$ for the data included in the fit. The values from the equation of state deviate somewhat from the experimental data but the relative deviations are within the tolerance of $2 \%$ associated with the EoS values. The three neglected data points of Banipal et al. [13] deviate by between $1.1 \%$ and $1.8 \%$.

TABLE 11. Parameters in the heat-capacity correlation, equation (19).

\begin{tabular}{cccc}
\hline$T_{0} / \mathrm{K}$ & $e_{0}$ & $e_{1}$ & $e_{2}$ \\
\hline 273.15 & 1530.400 & 328.251 & 275.245 \\
\hline
\end{tabular}

The thermodynamic integration was carried out on a regular grid extending from $p=(0.1$ to 390) $\mathrm{MPa}$ and from $T=(283.15$ to 473.15$) \mathrm{K}$ with mesh size given by $\delta p=0.1 \mathrm{MPa}$ and $\delta T=5 \mathrm{~K}$. The results of the thermodynamic integration at $T \leq 448.15 \mathrm{~K}$ are given on a reduced grid in Tables 12, 13, 14 and 15 for density, isothermal compressibility, isobaric expansivity and isobaric specific heat capacity, respectively.

To assess the likely uncertainties, we consider the sources of error associated with the initial values of density and isobaric specific heat capacity, and the sound-speed surface. A sensitivity analysis was carried out to estimate the effects of uncertainties in these inputs on the derived properties. Cosinusoidal temperature-dependent and, for the sound speed, pressure-dependent relative perturbations were considered such that the perturbed value $X_{\text {pert }}$ of an input quantity $X$ was given by

$$
X_{\text {pert }}=X\left[1+A \cdot \cos \left(k_{1} T\right) \cos \left(k_{2} p\right)\right] .
$$


TABLE 12. Derived densities $\rho$ of $n$-nonane as a function of temperature $T$ and pressure $p$.

\begin{tabular}{llllllllll}
\hline \multicolumn{1}{c}{$T / \mathrm{K}=$} & 283.15 & 298.15 & 323.15 & 348.15 & 373.15 & 398.15 & 423.15 & 448.15 \\
\hline$p / \mathrm{MPa}$ & & & & \multicolumn{2}{c}{$\rho /\left(\mathrm{kg} \cdot \mathrm{m}^{-3}\right)$} \\
0.1 & 725.55 & 713.99 & 694.37 & 674.19 & 653.31 & 631.54 & 608.58 & 584.04 \\
30 & 745.39 & 735.65 & 719.40 & 703.13 & 686.82 & 670.51 & 654.24 & 638.19 \\
60 & 761.20 & 752.62 & 738.38 & 724.19 & 710.09 & 696.12 & 682.39 & 669.09 \\
90 & 774.53 & 766.77 & 753.90 & 741.11 & 728.41 & 715.86 & 703.59 & 691.79 \\
120 & 786.16 & 779.02 & 767.19 & 755.41 & 743.72 & 732.18 & 720.90 & 710.07 \\
150 & 796.52 & 789.89 & 778.88 & 767.90 & 756.99 & 746.20 & 735.66 & 725.54 \\
180 & 805.90 & 799.70 & 789.37 & 779.05 & 768.76 & 758.58 & 748.62 & 739.04 \\
210 & 814.50 & 808.66 & 798.91 & 789.14 & 779.39 & 769.71 & 760.21 & 751.08 \\
240 & 822.46 & 816.93 & 807.69 & 798.40 & 789.10 & 779.84 & 770.74 & 761.96 \\
270 & 829.87 & 824.62 & 815.83 & 806.97 & 798.07 & 789.17 & 780.40 & 771.93 \\
300 & 836.81 & 831.82 & 823.44 & 814.96 & 806.41 & 797.83 & 789.35 & 781.14 \\
330 & 843.35 & 838.59 & 830.59 & 822.46 & 814.22 & 805.92 & 797.69 & 789.72 \\
360 & 849.54 & 845.00 & 837.34 & 829.53 & 821.57 & 813.53 & 805.52 & 797.76 \\
390 & 855.41 & 851.07 & 843.74 & 836.22 & 828.53 & 820.72 & 812.90 & 805.33 \\
\hline
\end{tabular}

TABLE 13. Derived isothermal compressibilities $\beta_{T}$ of $n$-nonane as a function of temperature $T$ and pressure $p$.

\begin{tabular}{lllllllll}
\hline \multicolumn{1}{c}{$T / \mathrm{K}=$} & 283.15 & 298.15 & 323.15 & 348.15 & 373.15 & 398.15 & 423.15 & 448.15 \\
\hline$p / \mathrm{MPa}$ & & & & \multicolumn{6}{c}{$\beta_{T} / \mathrm{GPa}^{-1}$} \\
0.1 & 1.0582 & 1.1853 & 1.4421 & 1.7744 & 2.2150 & 2.8169 & 3.6701 & 4.9370 \\
30 & 0.7813 & 0.8554 & 0.9901 & 1.1403 & 1.3087 & 1.4992 & 1.7176 & 1.9712 \\
60 & 0.6306 & 0.6801 & 0.7664 & 0.8575 & 0.9541 & 1.0572 & 1.1680 & 1.2868 \\
90 & 0.5329 & 0.5691 & 0.6309 & 0.6943 & 0.7597 & 0.8275 & 0.8976 & 0.9696 \\
120 & 0.4637 & 0.4918 & 0.5391 & 0.5869 & 0.6353 & 0.6845 & 0.7342 & 0.7838 \\
150 & 0.4116 & 0.4344 & 0.4723 & 0.5103 & 0.5483 & 0.5862 & 0.6239 & 0.6607 \\
180 & 0.3709 & 0.3899 & 0.4214 & 0.4527 & 0.4836 & 0.5141 & 0.5439 & 0.5728 \\
210 & 0.3380 & 0.3542 & 0.3811 & 0.4076 & 0.4335 & 0.4588 & 0.4831 & 0.5066 \\
240 & 0.3107 & 0.3250 & 0.3484 & 0.3713 & 0.3935 & 0.4148 & 0.4353 & 0.4548 \\
270 & 0.2878 & 0.3005 & 0.3212 & 0.3414 & 0.3607 & 0.3791 & 0.3965 & 0.4132 \\
300 & 0.2682 & 0.2796 & 0.2982 & 0.3162 & 0.3333 & 0.3494 & 0.3644 & 0.3788 \\
330 & 0.2512 & 0.2616 & 0.2785 & 0.2948 & 0.3100 & 0.3242 & 0.3374 & 0.3501 \\
360 & 0.2363 & 0.2459 & 0.2614 & 0.2762 & 0.2900 & 0.3027 & 0.3144 & 0.3256 \\
390 & 0.2231 & 0.2320 & 0.2464 & 0.2600 & 0.2726 & 0.2840 & 0.2944 & 0.3044 \\
\hline
\end{tabular}


TABLE 14. Derived isobaric expansivities $\alpha_{p}$ of $n$-nonane as a function of temperature $T$ and pressure $p$.

\begin{tabular}{lcccccccc}
\hline \multicolumn{1}{c}{$T / \mathrm{K}=$} & 283.15 & 298.15 & 323.15 & 348.15 & 373.15 & 398.15 & 423.15 & 448.15 \\
\hline$p / \mathrm{MPa}$ & & & & \multicolumn{7}{c}{$\alpha_{p} /\left(10^{-4} \mathrm{~K}^{-1}\right)$} \\
0.1 & 10.55 & 10.86 & 11.45 & 12.16 & 13.03 & 14.13 & 15.55 & 17.47 \\
30 & 8.45 & 8.71 & 9.07 & 9.33 & 9.53 & 9.68 & 9.81 & 9.97 \\
60 & 7.28 & 7.48 & 7.73 & 7.88 & 7.94 & 7.93 & 7.87 & 7.79 \\
90 & 6.46 & 6.63 & 6.83 & 6.94 & 6.96 & 6.91 & 6.80 & 6.66 \\
120 & 5.85 & 6.00 & 6.18 & 6.27 & 6.27 & 6.21 & 6.09 & 5.94 \\
150 & 5.35 & 5.50 & 5.67 & 5.75 & 5.76 & 5.69 & 5.58 & 5.43 \\
180 & 4.94 & 5.09 & 5.25 & 5.34 & 5.35 & 5.29 & 5.18 & 5.05 \\
210 & 4.59 & 4.74 & 4.91 & 5.00 & 5.01 & 4.96 & 4.87 & 4.74 \\
240 & 4.28 & 4.43 & 4.61 & 4.70 & 4.73 & 4.69 & 4.60 & 4.49 \\
270 & 4.01 & 4.16 & 4.35 & 4.45 & 4.48 & 4.46 & 4.38 & 4.27 \\
300 & 3.76 & 3.92 & 4.12 & 4.23 & 4.27 & 4.26 & 4.19 & 4.09 \\
330 & 3.53 & 3.70 & 3.91 & 4.03 & 4.09 & 4.08 & 4.02 & 3.93 \\
360 & 3.32 & 3.50 & 3.72 & 3.86 & 3.92 & 3.92 & 3.87 & 3.79 \\
390 & 3.12 & 3.31 & 3.54 & 3.70 & 3.77 & 3.78 & 3.74 & 3.66 \\
\hline
\end{tabular}

TABLE 15. Derived isobaric specific heat capacities $c_{p}$ of $n$-nonane as a function of temperature $T$ and pressure $p$.

\begin{tabular}{lllllllll}
\hline \multicolumn{1}{c}{$T / \mathrm{K}=$} & 283.15 & 298.15 & 323.15 & 348.15 & 373.15 & 398.15 & 423.15 & 448.15 \\
\hline$p / \mathrm{MPa}$ & & & & \multicolumn{7}{c}{$c_{p} /\left(\mathrm{J} \cdot \mathrm{kg}^{-1} \cdot \mathrm{K}^{-1}\right)$} \\
0.1 & 2166 & 2217 & 2304 & 2396 & 2493 & 2594 & 2700 & 2810 \\
30 & 2133 & 2181 & 2265 & 2353 & 2443 & 2534 & 2622 & 2703 \\
60 & 2108 & 2157 & 2243 & 2333 & 2426 & 2519 & 2608 & 2685 \\
90 & 2088 & 2138 & 2227 & 2321 & 2417 & 2514 & 2606 & 2684 \\
120 & 2070 & 2123 & 2215 & 2312 & 2412 & 2513 & 2608 & 2688 \\
150 & 2055 & 2109 & 2204 & 2304 & 2409 & 2513 & 2612 & 2694 \\
180 & 2041 & 2096 & 2194 & 2298 & 2406 & 2514 & 2616 & 2700 \\
210 & 2027 & 2084 & 2185 & 2292 & 2403 & 2515 & 2620 & 2705 \\
240 & 2014 & 2072 & 2176 & 2286 & 2401 & 2516 & 2624 & 2711 \\
270 & 2001 & 2061 & 2167 & 2280 & 2398 & 2516 & 2627 & 2716 \\
300 & 1988 & 2049 & 2158 & 2274 & 2395 & 2516 & 2630 & 2720 \\
330 & 1974 & 2037 & 2148 & 2267 & 2392 & 2516 & 2632 & 2725 \\
360 & 1961 & 2025 & 2139 & 2261 & 2388 & 2516 & 2635 & 2729 \\
390 & 1946 & 2012 & 2128 & 2254 & 2384 & 2515 & 2637 & 2733 \\
\hline
\end{tabular}


Here, $A$ is an amplitude and $k_{1}$ and $k_{2}$ determine the wavelengths of the perturbation in temperature and pressure. For the initial density and heat capacity, pressure is not a relevant variable and $k_{2}=0$. The amplitudes $A$ considered were $0.05 \%$ for density, $0.5 \%$ for isobaric specific heat capacity and $0.1 \%$ for sound speed, based approximately on the estimated expanded relative uncertainties of each quantity. Numerous scenarios were investigated; Table 16 exemplifies the results and additional graphical examples are given in the Supplementary Material for the case of sound-speed perturbations. In each of these scenarios, either an initial value function or the sound-speed surface were perturbed separately and the absolute average and absolute maximum relative deviations from the unperturbed case were calculated. As observed in previous studies [ $42 ; 47 ; 49 ; 50]$, constant perturbations to the initial values of either $\rho$ or $c_{p}\left(k_{1}=0\right)$ were found to cause perturbation to the derived properties that tended to decay with increasing pressure. When $k_{1}$ was increased from zero, the resulting perturbations were observed to grow with increasing pressure, especially near the lower temperature limit, with the largest relative effect being observed for $\alpha_{p}$. At $k_{1}=0.05 \mathrm{~K}^{-1}$ (corresponding to a wavelength of $125 \mathrm{~K}$ ), the maximum errors were almost $0.1 \%$ in $\rho$ and $0.8 \%$ in $c_{p}$. Given the form of the smoothing equations used to represent $\rho$ and $c_{p}$ on the initial isobar, shorter wavelength oscillatory errors seem unlikely. When the sound speed surface is perturbed, the resulting errors in the derived properties increase with pressure. The worst case is when $k_{2}=0$; the effects on the derived values of $\rho$ remain small but much larger relative errors in $\alpha_{p}$ are observed, reaching almost $3 \%$ in the worst case.

TABLE 16. Effects of perturbations to the initial values and the sound-speed surface used in thermodynamic integration, where $\mathrm{X}$ denotes the property perturbed and $A, k_{1}$ and $k_{2}$ are the parameters of the cosinusoidal perturbation functions.

\begin{tabular}{ccccccccc|cccc|}
\hline & & & & \multicolumn{5}{c|}{$10^{2} \Delta_{\mathrm{AARD}}$} & \multicolumn{4}{c|}{$10^{2} \Delta_{\mathrm{MARD}}$} \\
$X$ & $10^{2} A$ & $k_{1} / \mathrm{K}^{-1}$ & $k_{2} / \mathrm{MPa}^{-1}$ & $\rho$ & $b_{T}$ & $\alpha_{p}$ & $c_{p}$ & $\rho$ & $b_{T}$ & $\alpha_{p}$ & $c_{p}$ \\
\hline$\rho$ & 0.05 & 0 & - & 0.04 & 0.02 & 0.1 & 0.01 & 0.05 & 0.08 & 0.3 & 0.03 \\
$\rho$ & 0.05 & 0.05 & - & 0.03 & 0.38 & 2.1 & 1.1 & 0.16 & 1.68 & 12.4 & 3.9 \\
$c_{p}$ & 0.5 & 0 & - & 0.01 & 0.04 & 0.1 & 0.5 & 0.01 & 0.10 & 0.3 & 0.6 \\
$c_{p}$ & 0.5 & 0.05 & - & 0.01 & 0.07 & 0.5 & 0.5 & 0.04 & 0.58 & 3.8 & 1.5 \\
$c$ & 0.1 & 0 & - & 0.01 & 0.15 & 0.1 & 0.01 & 0.02 & 0.18 & 0.29 & 0.03 \\
$c$ & 0.1 & 0.05 & - & 0.01 & 0.09 & 0.5 & 0.3 & 0.04 & 0.71 & 4.9 & 1.6 \\
$c$ & 0.1 & 0.05 & 0.02 & 0.00 & 0.09 & 0.2 & 0.1 & 0.02 & 0.24 & 1.4 & 0.4 \\
\hline
\end{tabular}

A partial validation of the results of the thermodynamic integration was obtained by comparing the derived values of density with those directly measured. For this purpose, cubic interpolation was used to permit comparison at identical pressures. Fig. 7 compares the 
experimental compressed-liquid densities with those obtained by thermodynamic integration. All relative deviations fall between $-0.09 \%$ and $0.06 \%$.

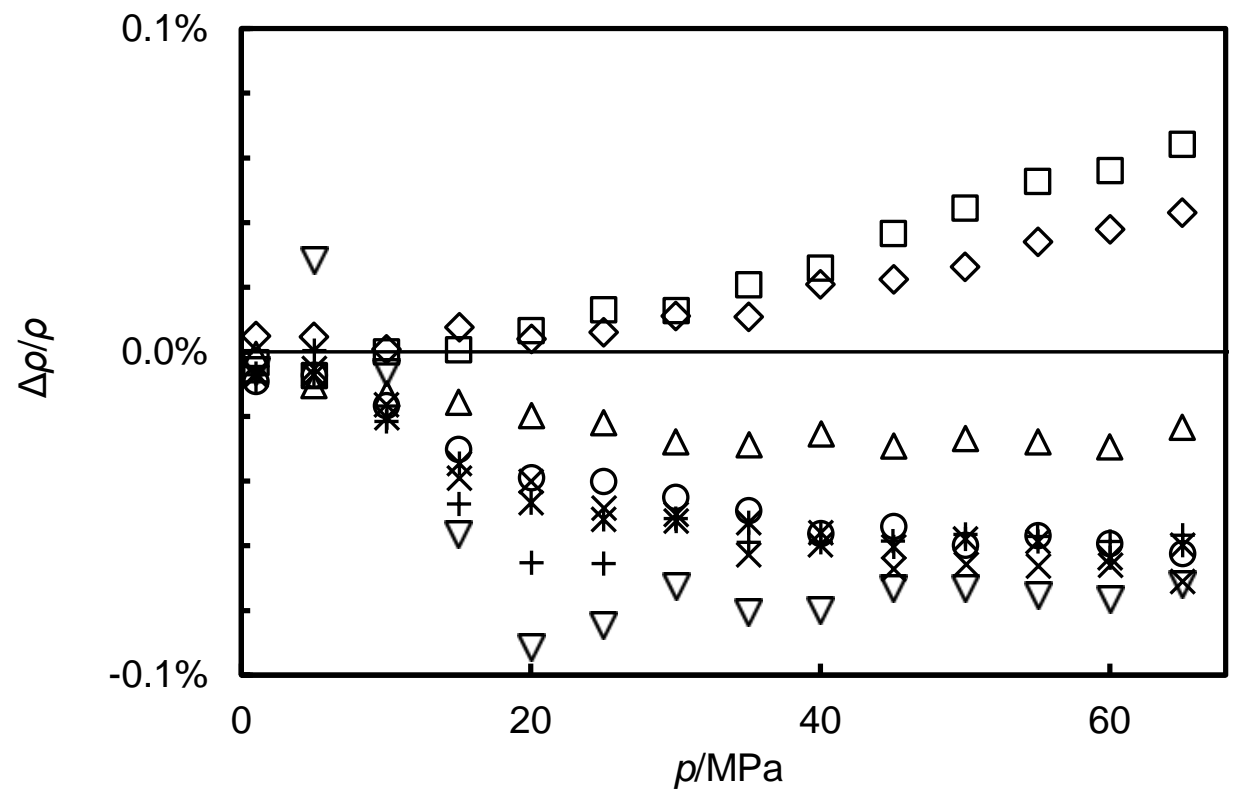

Fig. 7. Relative deviations $\Delta \rho / \rho=\left(\rho-\rho_{\text {Int }}\right) / \rho$ of experimental densities $\rho$ of $n$-nonane from the values $\rho_{\text {Int }}$ obtained by thermodynamic integration: $\square, T=283.15 \mathrm{~K} ; \diamond, T=298.15 \mathrm{~K} ; \triangle$, $T=323.15 \mathrm{~K} ; O, T=348.15 \mathrm{~K} ; \times, T=373.15 \mathrm{~K} ; *, T=398.15 \mathrm{~K} ; \quad+, T=423.15 \mathrm{~K} ; \nabla$, $T=448.15 \mathrm{~K}$.

We also recognise that a crucial step in the thermodynamic integration is the calculation of the first two temperature derivatives of $\rho$ along each isobar. In our algorithm, this is accomplished by fitting a smoothing equation to $\rho$ as a function of $T$ on each isobar as the solution is constructed in pressure increments. The smoothing equation used was in the form of Eq. (11) with reduced temperature $t$ as the independent variable raised to powers of $0,1 / 3,2$ and 4 . Replacing this procedure with numerical differentiation based on a small number of points leads to numerical instability and the rapid growth of parasitic solutions. Therefore, some degree of smoothing appears to be essential. As a further sensitivity test, the thermodynamic integration was repeated with a simple cubic equation in reduced temperature applied on all isobars:

$$
\rho_{0} /\left(\mathrm{kg} \cdot \mathrm{m}^{-3}\right)=a_{0}^{\prime}+a_{1}^{\prime} \tau+a_{2}^{\prime} \tau^{2}+a_{4}^{\prime} \tau^{3}
$$

The initial coefficients of this polynomial for the isobar at $p_{0}=0.1 \mathrm{MPa}$ were obtained by matching to equation (11) with coefficients from Table 8, yielding $a_{0}^{\prime}=393.926, a_{1}^{\prime}=975.512$, $a_{2}^{\prime}=-983.037$ and $a_{3}^{\prime}=629.736$. This correlation matches Eq. (11) to with $\pm 0.16 \mathrm{~kg} \cdot \mathrm{m}^{-3}$ and leads to an only slightly inferior representation of the experimental density data. When this 
function was used in the thermodynamic integration, it was found that the derived properties at high temperatures and/or low pressures were essentially unchanged. However, moving toward the highest pressure and the lowest temperature considered, differences of increasing magnitude are found reaching, in the worst case, $0.4 \%$ for $\rho$ and $9 \%$ for $c_{p}$. We conclude that the assumptions made in representing $\rho(T)$ along each isobar is a more important factor than plausible errors in either the initial conditions or the sound-speed surface. We can also identify a region of high pressure and low temperature within which the results are sensitive to the choice of smoothing function. In the present case, this is above an approximately straight line on the $(T, p)$ diagram extending from $p=165 \mathrm{MPa}$ at $T=283 \mathrm{~K}$ to $p=390 \mathrm{MPa}$ at about $T=348 \mathrm{~K}$.

From the sensitivity analyses conducted, we believe that it is possible to specify relative uncertainty bounds for the derived properties in the region $p / \mathrm{MPa} \leq \operatorname{Min}[165+3(T / \mathrm{K}-283.15)$, 390] as follows: $U_{\mathrm{r}}(\rho) \leq 0.1 \%, U_{\mathrm{r}}\left(\beta_{T}\right) \leq 1.5 \%, U_{\mathrm{r}}\left(\alpha_{p}\right) \leq 5 \%$ and $U_{\mathrm{r}}\left(c_{p}\right) \leq 2 \%$. However, it is not possible to attach a definite statistical significance to these values. The uncertainties are larger in the top-left of the $(T, p)$ diagram.

\section{Literature Comparison}

\subsection{Comparison of experimental data with literature data and models}

Figs 8 and 9 compare the experimentally-determined density and sound speed with the equation of state of Lemmon and Span [7]. The experimental densities are mostly slightly lower than predicted by the EoS model, with the worst deviation approaching $-0.3 \%$ at the higher temperatures and lower pressures studied. However, most of the data fall within about $0.1 \%$ of the EoS predictions, which is within the tolerance of the latter. 


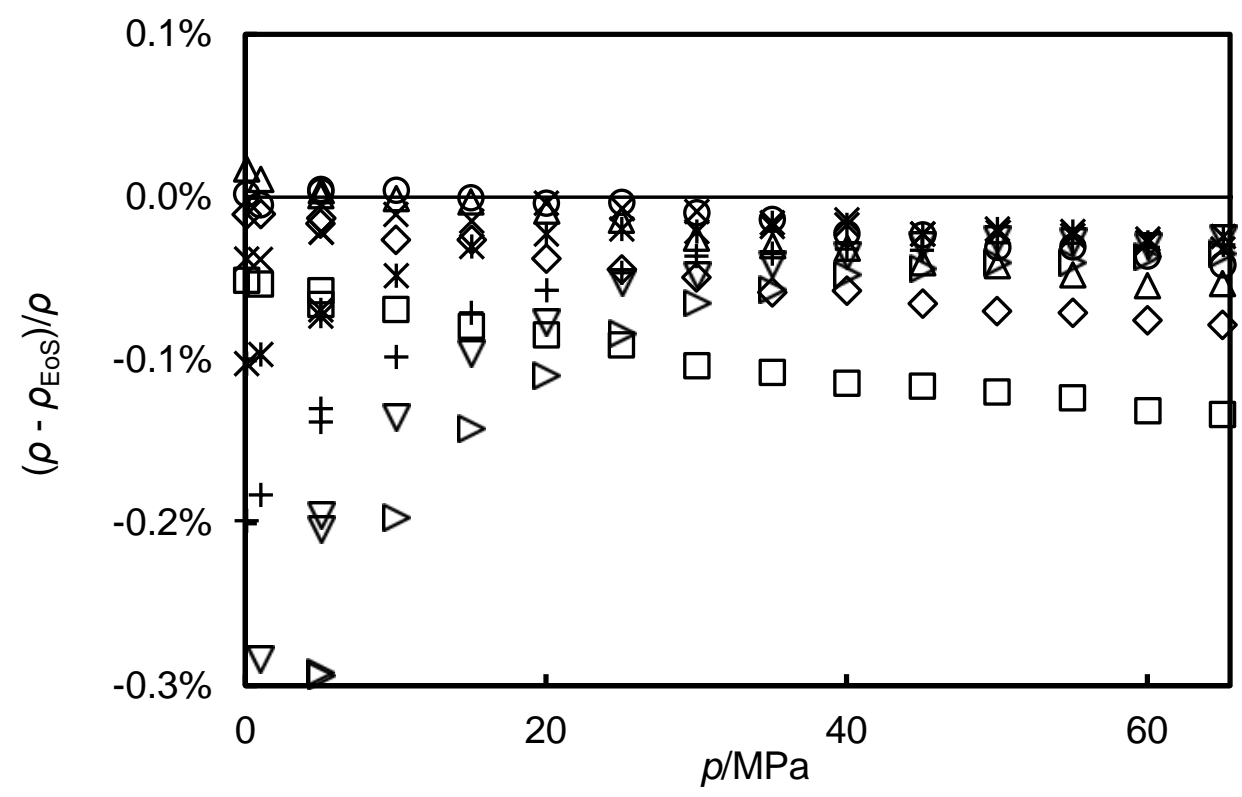

Fig. 8. Relative deviations $\left(\rho-\rho_{\text {Eos }}\right) / \rho$ of experimental densities $\rho$ of $n$-nonane from the values $\rho_{\text {EoS }}$ obtained from the equation of state of Lemmon and Span [7]: $\square, T=283.15 \mathrm{~K} ; \diamond$, $T=298.15 \mathrm{~K} ; \triangle, T=323.15 \mathrm{~K} ; O, T=348.15 \mathrm{~K} ; \times, T=373.15 \mathrm{~K} ; *, T=398.15 \mathrm{~K} ;+$, $T=423.15 \mathrm{~K} ; \nabla, T=448.15 \mathrm{~K} ; \nabla, T=473.15 \mathrm{~K}$.

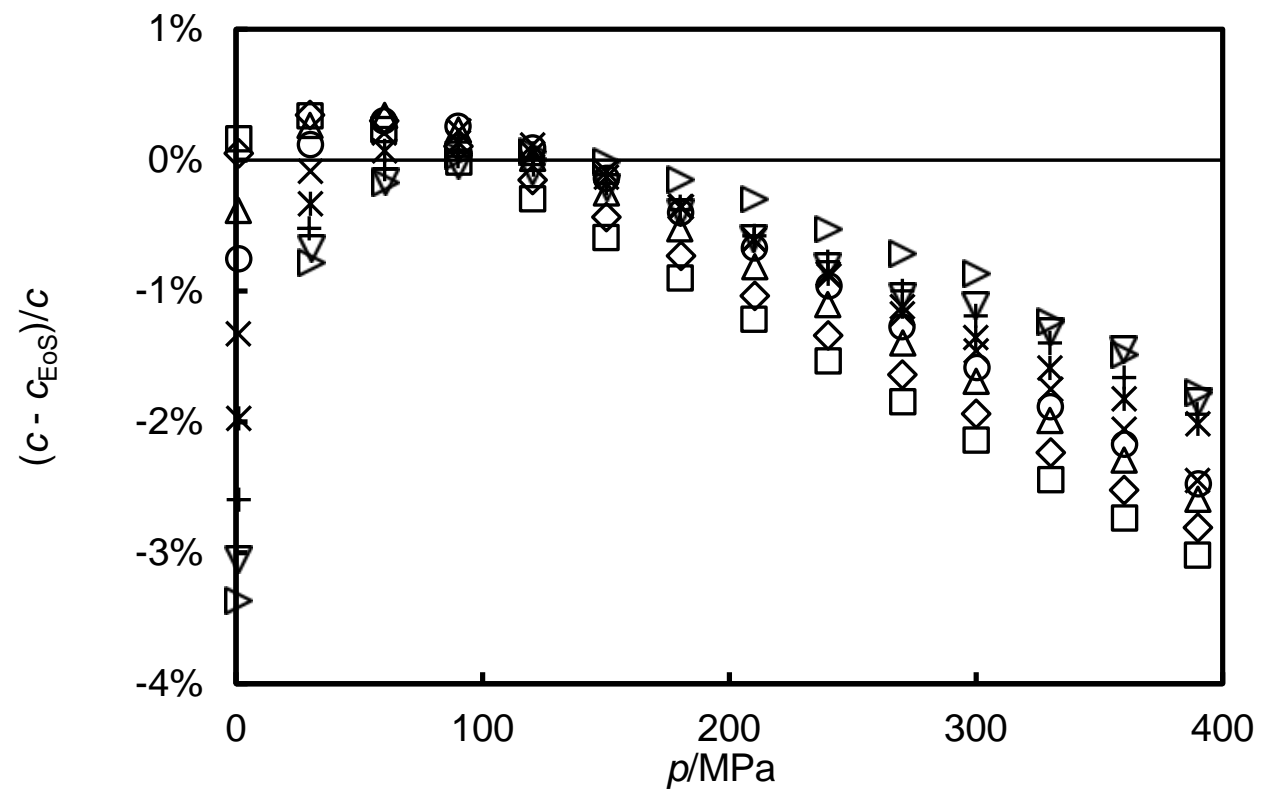

Fig. 9. Relative deviations $\left(c-c_{\text {Eos }}\right) / c$ of experimental speed of sound $c$ in $n$-nonane from the values $C_{\text {Eos }}$ obtained from the equation of state of Lemmon and Span [7]: $\square, T=283.15 \mathrm{~K} ; \diamond$, $T=298.15 \mathrm{~K} ; \triangle, T=323.15 \mathrm{~K} ; O, T=348.15 \mathrm{~K} ; \quad \times, T=373.15 \mathrm{~K} ; *, T=398.15 \mathrm{~K} ;+$, $T=423.15 \mathrm{~K} ; \nabla, T=448.15 \mathrm{~K} ; \nabla, T=473.15 \mathrm{~K}$.

The sound-speed data extend to much higher pressures and form a more severe test of the EoS. As seen in Fig. 9, the relative deviations of our experimental data from the equation of state of Lemmon and Span [7] are mostly within $\pm 0.5 \%$ at pressures between (30 and 150) MPa. However, sharply increasing deviations are seen at low pressures and high 
temperatures and, for all temperatures, at $p>200 \mathrm{MPa}$. The deviations observed at low pressures correlate to a certain extent with the deviations seen in the density.
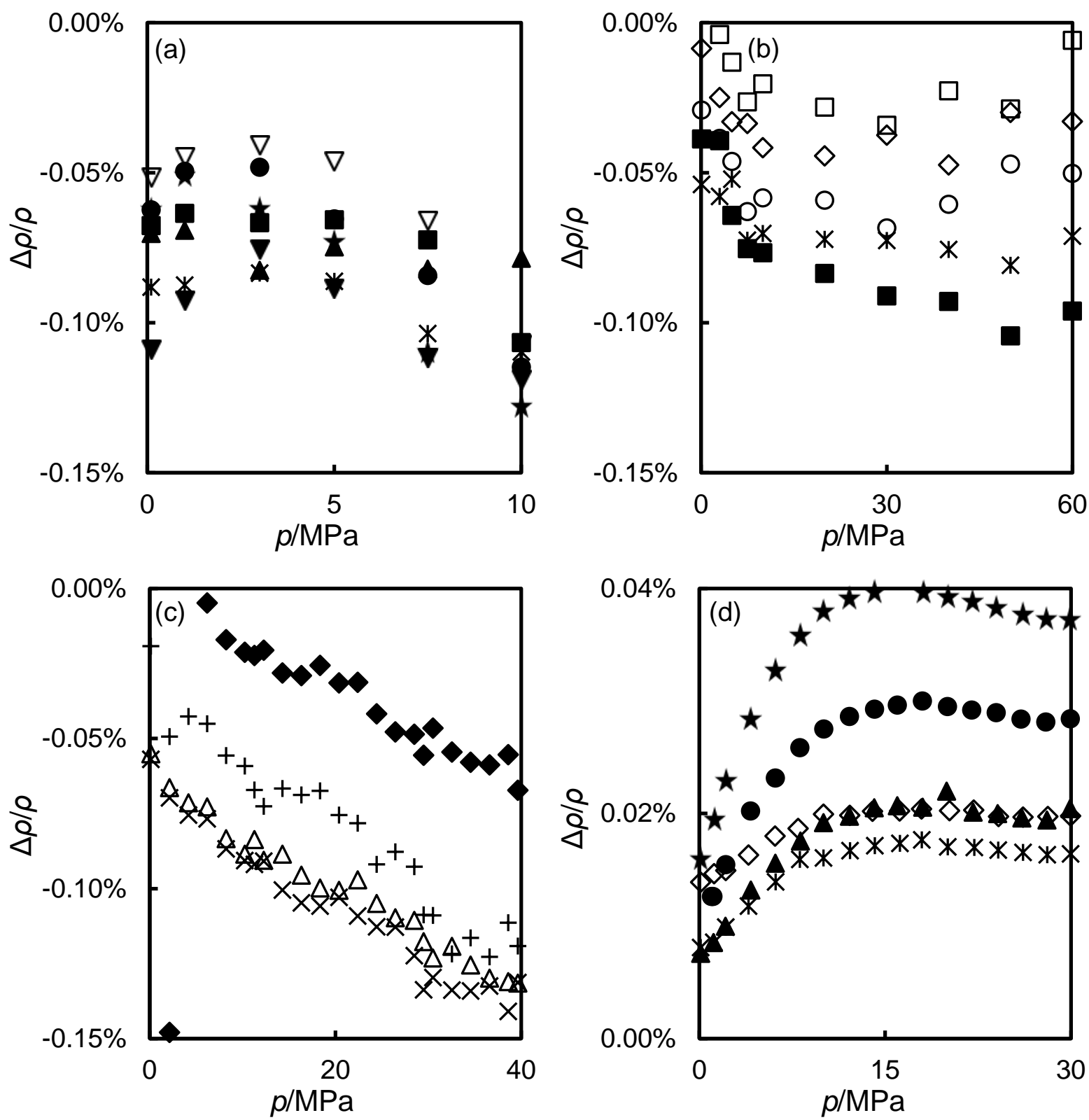

Fig. 10. Relative deviations $\Delta \rho / \rho=\left(\rho-\rho_{\text {calc }}\right) / \rho$ of literature densities $\rho$ of $n$-nonane from the values $\rho_{\text {calc }}$ calculated from Eq. (10) with parameters from Table 8. (a) Banipal et al. [13]; (b) Valencia et al. [15]; (c) Moravkova et al. [16]; (d) Schilling et al. [17]. Symbols: $\square$, $T=283.15 \mathrm{~K} ; \quad \diamond, T=293.15 \mathrm{~K} ; \quad \triangle, T=298.15 \mathrm{~K} ; \quad O, T=303.15 \mathrm{~K} ; \quad \times, T=308.15 \mathrm{~K}$; *, $T=313.15 \mathrm{~K} ; \quad+, T=318.15 \mathrm{~K} ; \mathbf{\square}, T=323.15 \mathrm{~K} ; \bullet, T=328.15 \mathrm{~K} ; \boldsymbol{\Delta}$, $T=333.15 \mathrm{~K} ; \quad \nabla, T=343.15 \mathrm{~K} ; \quad \bullet, T=353.15 \mathrm{~K} ; \nabla, T=363.15 \mathrm{~K} ; \quad \star \quad T=373.15 \mathrm{~K}$ 

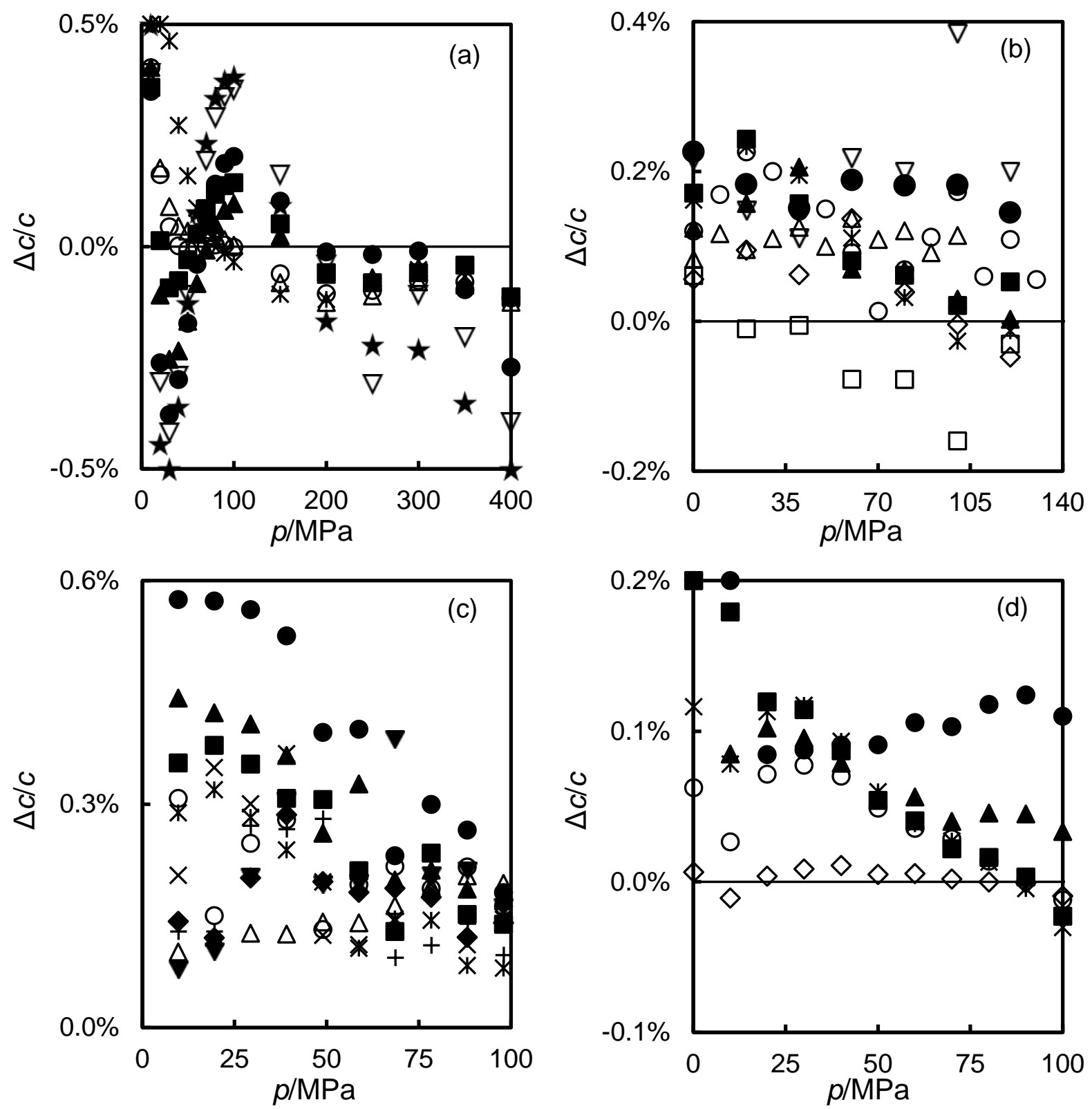

Fig. 11. Relative deviations $\Delta c / c=\left(c-c_{\text {calc }}\right) / c$ of the literature speeds of sound $c$ in $n$-nonane from the values $c_{\text {calc }}$ calculated from Eqs. (16) and (17) with parameters from Table 10. (a) Zotov et al. [21]; (b) Boelhouwer [19]; (c) Badalyan et al. [20]; (d) Lago et al. [22]. Symbols: $\square, T=273 \mathrm{~K} ; \diamond T=293 \mathrm{~K} ; \triangle, T=303 \mathrm{~K} ; O, T=313 \mathrm{~K} ; \times, T=323 \mathrm{~K} ; *, T=333 \mathrm{~K} ;+$, $T=343 \mathrm{~K} ; \boldsymbol{\square}, T=353 \mathrm{~K} ; \bullet, T=363 \mathrm{~K} ; \boldsymbol{\Delta}, T=373 \mathrm{~K} ; \boldsymbol{\nabla}, T=383 \mathrm{~K} ; \boldsymbol{\bullet}, T=393 \mathrm{~K} ; \nabla$, $T=413 \mathrm{~K} ; \star, T=433 \mathrm{~K}$

Fig. 10 compares literature density data with the modified Tait equation that fits the present experimental data to within $\pm 0.02 \%$. We can observe that the vibrating-tube data of Banipal et al. [13], Valencia et al. [15], and Moravkova et al. [16] all exhibit small but significant negative deviations from our Tait equation. The best agreement observed is with the data of Schilling et al. [17] which are associated with an expanded uncertainty of only $0.02 \%$. As seen in Fig. 10(d), small systematic positive deviations are observed which average $0.02 \%$. These are of 
the same sign and are similar in magnitude to the deviations observed when our methylbenzene data were compared with the reference correlation of McLinden and Splett [40] (note that the sign of $\Delta \rho$ is reversed between Figs. 2 and 10). Nevertheless, the agreement with Schilling et al. is within the combined uncertainty.

To compare the present sound-speed data with experimental values from the literature, we make use of our surface fit, Eqs. (16) and (17) with parameters from Table 10, as a baseline and plot deviations of the literature data from this in Fig. 11. The data of Zotov et al. [21] mostly fall within $\pm 0.5 \%$ and are evenly distributed about the zero line. The other three sources mostly show small positive biases with respect to our surface fit: about $0.1 \%$ for Boelhouwer [19], $0.2 \%$ for Badalyan et al. [20] and $0.1 \%$ for Lago et al. [22]; however, all of these are within the combined uncertainties. The data of Lago et al. were measured with an instrument similar to the one used in this work and are associated with an overall relative uncertainty of $\leq 0.2 \%$. We note that three of their data points deviate substantially from our surface fit. These authors commented that their measurement at $T=393.15 \mathrm{~K}$ and $p=0.1 \mathrm{MPa}$ was considered unreliable and this point is found to deviate by $2.9 \%$ from our surface fit. The other two apparently discrepant points are at (373.15 K, $0.1 \mathrm{MPa})$ and $(393.15 \mathrm{~K}, 10 \mathrm{MPa})$, both of which exhibit deviations of $\Delta c / c=0.7 \%$. Otherwise, agreement with the results of Lago et al. is well within the combined uncertainty.

\subsection{Comparison of derived properties with literature data}

We next compare the derived properties, obtained in this work by thermodynamic integration, with data from the literature. In order to facilitate this, bicubic interpolations were performed on the grid of calculated properties thereby permitting comparison with literature data that did not coincide with the calculated grid. This procedure is estimated to introduce negligible additional uncertainty.

Fig. 12 compares the available compressed-liquid heat capacity data with the calculated thermodynamic surface. The values shown at $p=0.1 \mathrm{MPa}$ are those obtained by extrapolation for use in the development of Eq. (19) and the deviations are identical to those shown in Fig . 6(b). Fig. 12(a) shows the data of Banipal et al. which exhibit deviation of between $-0.4 \%$ and $2.3 \%$, compared with the stated uncertainties of about $0.5 \%$. At temperatures up to $358.15 \mathrm{~K}$, the deviations are within the range $-0.4 \%$ to $1.2 \%$ and we note that the deviations do not vary much with pressure over the range investigated. The three isotherms at $T \geq 363.15 \mathrm{~K}$ are those rejected in the determination of the parameters of Eq. (19). In Fig. 12(b), we show the data of Kuznetsov et al. [18] and these deviate from our thermodynamic surface by 
between $-0.1 \%$ and $1.3 \%$. The deviations increase from near zero at $p=0.1 \mathrm{MPa}$ and appear to level out at pressures above about $30 \mathrm{MPa}$. Overall, the heat capacity data appear to be in fair agreement with our thermodynamic surface. The two available data sets are, at the level of $1 \%$, slightly inconsistent in the overlapping regions of temperature and pressure.
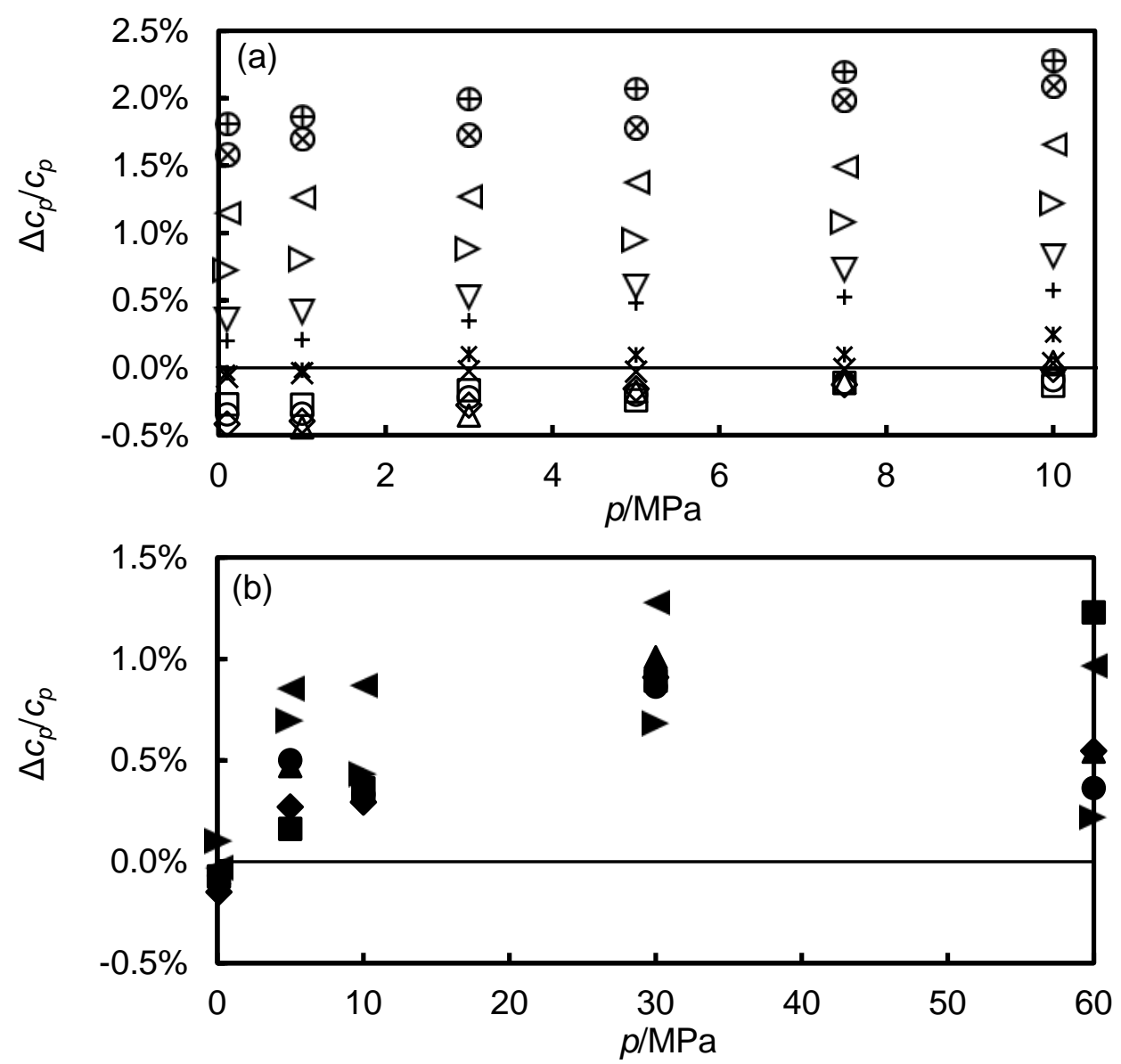

Fig. 12. Relative deviation $\Delta c_{p} / c_{p}=\left(c_{p}-c_{p, \mathrm{Int}}\right) / c_{p}$ of literature isobaric specific heat capacities $c_{p}$ from the values $c_{p, \text { Int }}$ determined by thermodynamic integration. (a) Banipal et al. [13]: $\square, T=318.15 \mathrm{~K} ; \diamond, T=32.153 \mathrm{~K} ; \triangle, T=328.15 \mathrm{~K} ; \quad O, T=333.15 \mathrm{~K} ; \quad \times, T=338.15 \mathrm{~K}$; *, $T=343.15 \mathrm{~K} ; \quad+, T=348.15 \mathrm{~K} ; \nabla, T=353.15 \mathrm{~K} ; \quad \nabla, T=358.15 \mathrm{~K}$; $\triangleleft, T=363.15 \mathrm{~K} ; \otimes, T=368.15 \mathrm{~K} ; \oplus, T=373.15 \mathrm{~K}$. (b) Kuznetsov et al. [18]: $\boldsymbol{\square}, T=323.9 \mathrm{~K} ; \quad \bullet, T=355.9 \mathrm{~K} ; \quad \boldsymbol{\Delta}, T=375.2 \mathrm{~K} ; \quad \boldsymbol{\theta}, T=403.5 \mathrm{~K} ; \quad \boldsymbol{}, T=429.6 \mathrm{~K} ;$ $4, T=459.4 \mathrm{~K}$.

Fig. 13 compares the available high-pressure density and relative volume data with the calculated thermodynamic surface at temperatures from $298.15 \mathrm{~K}$ to $423.15 \mathrm{~K}$. Overall, the relative deviations span the range from $-0.3 \%$ to $0.4 \%$. The data of Doolittle [10] and Grindley and Lind [11] show mostly positive deviations, while those of Scaife and Lyons [12] and Boelhouwer [9] exhibit the opposite trend. This correlates with the inconsistency noted by Lemmon and Span [7]; the present data pass in between these sets. We also note that the 
density data of Scaife and Lyons [12] and the relative volumes of Boelhouwer [9] have somewhat different trends with temperature; the former deviates more at low temperatures while the latter shows larger deviations at high temperatures. The data of Brostow et al. [14] are almost identical to those of Grindley and Lind [11] and so are not shown in Fig. 13. Overall, our calculated thermodynamic surface does not strongly favour any one of the literature data sets.
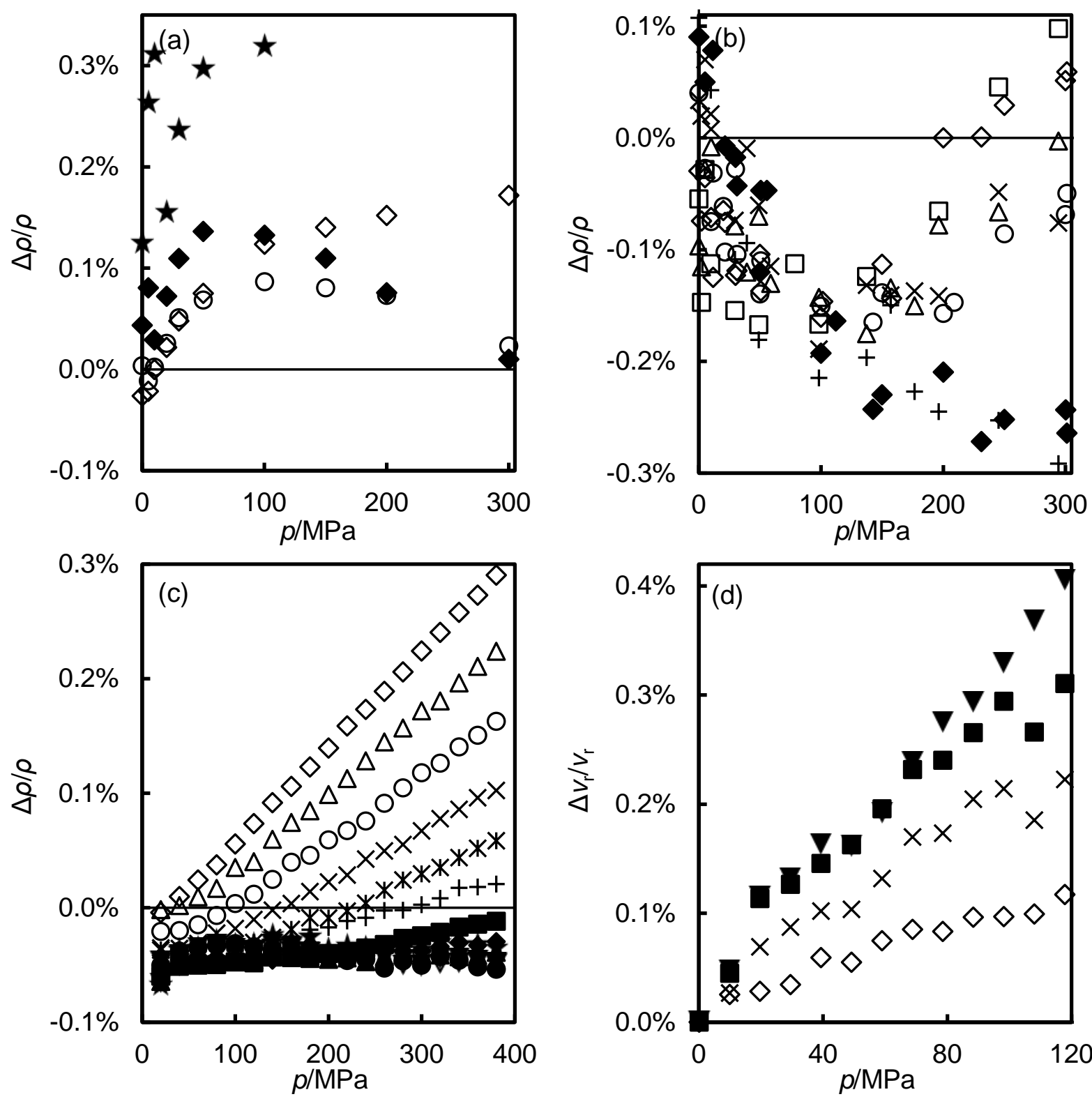

Fig. 13. Relative deviations $\Delta \rho / \rho=\left(\rho-\rho_{\text {calc }}\right) / \rho$ and $\Delta v_{r} / v_{r}=\left(v_{r}-v_{r}\right.$,calc $) / v_{r}$ of literature densities $\rho$ or relative volumes $v_{\mathrm{r}}$ of $n$-nonane from the values $\rho_{\text {calc }}$ or $v_{\mathrm{r}}$ obtained by thermodynamic integration. (a) Doolittle et al. [10]; (b) Scaife and Lyonds [12]; (c) Grindley et al. [11]; (d) Boelhouwer [9]. Symbols: $\square, T=298.15 \mathrm{~K} ; \diamond, T=303.15 \mathrm{~K} ; \triangle, T=313.15 \mathrm{~K} ; \mathrm{O}$, $T=323.15 \mathrm{~K} ; \times, T=333.15 \mathrm{~K} ; *, T=343.15 \mathrm{~K} ;+, T=353.15 \mathrm{~K} ; \boldsymbol{\square}, T=363.15 \mathrm{~K} ; \bullet$, $T=373.15 \mathrm{~K} ; \boldsymbol{\Delta}, T=383.15 \mathrm{~K} ; \boldsymbol{\nabla}, T=393.15 \mathrm{~K} ; \boldsymbol{\bullet}, T=403.15 \mathrm{~K} ; \nabla, T=413.15 \mathrm{~K} ; \star$, $T=423.15 \mathrm{~K}$. 
The lower-pressure density of Banipal et al. [13], Valencia et al. [15], and Moravkova et al. [16] show deviations from the thermodynamic surface that are very similar to those from the Tait equation shown in Fig. 10. It is however notable that the very accurate date of Schilling et al. [17] have smaller deviations from the thermodynamic surface than shown in Fig. 10(d), with $\Delta_{\text {AARD }}=0.011 \%$.

\section{Conclusions}

The present work is a comprehensive study of the properties of $n$-nonane at pressures up to $390 \mathrm{MPa}$ and temperatures from $283 \mathrm{~K}$ to $473 \mathrm{~K}$. The experimental data will be useful in the future development of an improved wide-range equation of state. Several conclusions can be drawn concerning the methods of measurement and analysis. A careful and thorough analysis of uncertainty shows that type-A uncertainties in the density are extremely small: for example, about $0.07 \mathrm{~kg} \cdot \mathrm{m}^{-3}$ at the median state point where $\rho=686 \mathrm{~kg} \cdot \mathrm{m}^{-3}$. However, comparison of our validation measurements with very accurate reference densities for methylbenzene show deviations averaging $0.25 \mathrm{~kg} \cdot \mathrm{m}^{-3}$ and reaching $0.41 \mathrm{~kg} \cdot \mathrm{m}^{-3}$ in the worst case. This suggests that the working equation is not quite compatible with the data over the full range from the density of helium ( $<100 \mathrm{~kg} \cdot \mathrm{m}^{-3}$ at all state points) to that of water. Calibration including fluids having densities more similar to those of the test fluid would therefore be advantageous; unfortunately, density standards of the necessary low uncertainty are not available for the entire ranges of temperature and pressure investigated in this work. We also draw conclusions concerning the method of thermodynamic integration and the reliability of the results obtained. Sensitivity analyses show that the thermodynamic surface obtained by this method is generally very robust in the face of small uncertainties in the input quantities, except towards the topleft of the temperature-pressure diagram where the effects of such errors are found to become increasingly significant. The present study extends over a wider range of temperature than previously considered and we find that the results also have some sensitivity to the way in which the temperature derivatives of density are calculated along each isobar. Different smoothing equations lead to slightly different results and these effects are most pronounced at low temperatures and high pressures. Improved numerical methods might be developed in the future to address this problem.

\section{Acknowledgement}

This work was supported by the Engineering and Physical Sciences Research Council through a Doctoral Training Award. 


\section{References}

[1] E.C. Ihmels, J. Gmehling, Ind. Eng. Chem. Res. 40 (2001) 4470-4477.

[2] H. Li, J.P. Jakobsen, Ø. Wilhelmsen, J. Yan, Appl. Energy 88 (2011) 3567-3579.

[3] J.L. Daridon, B. Lagourette, J.F. Gaubert, P. Xans, F. Montel, Ultrasonics 34 (1996) 447-449.

[4] D.-Y. Peng, D.B. Robinson, Ind. Eng. Chem. Fundam. 15 (1976) 59-64.

[5] G. Soave, Chem. Eng. Sci. 27 (1972) 1197-1203.

[6] O. Kunz, W. Wagner, J. Chem. Eng. Data 57 (2012) 3032-3091.

[7] E.W. Lemmon, R. Span, J. Chem. Eng. Data 51 (2006) 785-850.

[8] L.T. Carmichael, B.H. Sage, W. Lacy, Ind. Eng. Chem. 45 (1953) 2697-2699.

[9] J.W.M. Boelhouwer, Physica 26 (1960) 1021-1028.

[10] A.K. Doolittle, J. Chem. Eng. Data 9 (1964) 275-279.

[11] T. Grindley, J.E. Lind, J. Chem. Phys. 68 (1978) 5046-5052.

[12] W.G.S. Scaife, C.G.R. Lyonds, Proc. Roy. Soc. London. A Mat. 370 (1980) 193-211.

[13] T.S. Banipal, S.K. Garg, J.C. Ahluwalia, J. Chem. Thermodyn. 23 (1991) 923-931.

[14] W. Brostow, T. Grindley, M.A. Macip, Mater. Chem. Phys. 12 (1985) 37-97.

[15] J.L. Valencia, D. Gonzalez-Salgado, J. Troncoso, J. Peleteiro, E. Carballo, L. Romani, J. Chem. Eng. Data 54 (2009) 904-915.

[16] L. Morávková, Z. Wagner, J. Linek, J. Chem. Thermodyn. 39 (2007) 1637-1648.

[17] G. Schilling, R. Kleinrahm, W. Wagner, J. Chem. Thermodyn. 40 (2008) 1095-1105.

[18] M.A.K. Kuznetsov, V.E.; Gerasimov, A.A.; Grigor'ev, M.D., Neft i Gaz 31 (1988).

[19] J.W.M. Boelhouwer, Physica 34 (1967) 484-492.

[20] A.L. Badalyan, N.F. Otpushchennikov, Y.S. Shoitov, Uch. Zap. Kursk. Gos. Pedagog. Inst. 91 (1971) 135-143.

[21] M.Y.F. Zotov V V, Mel'nikov G A, Neruchev Yu A, Sound Velocity in Liquid Hydrocarbons, Kursk State Pedagogical University, Kursk, 1995.

[22] S. Lago, P.A.G. Albo, D.M. Ripa, Int. J. Thermophys. 27 (2006) 1083-1094.

[23] L.M. Casas, B. Orge, J. Tojo, J. Chem. Eng. Data 49 (2004) 664-667.

[24] S.M. Pereira, M.A. Rivas, J.N. Real, J.L. Legido, T.P. Iglesias, J. Chem. Eng. Data 47 (2002) 919-922.

[25] T.M. Aminabhavi, B. Gopalkrishna, J. Chem. Eng. Data 39 (1994) 529-534.

[26] L. Andreoli-Ball, D. Patterson, M. Costas, M. Caceresalonso, J. Chem. Soc., Faraday Trans. 84 (1988) 3991-4012.

[27] J.P.E. Grolier, M.H. Hamedi, E. Wilhelm, H.V. Kehiaian, Thermochim. Acta 31 (1979) 79-84.

[28] E. Calvo, P. Brocos, R. Bravo, M. Pintos, A. Amigo, A.H. Roux, G. Roux-Desgranges, J. Chem. Eng. Data 43 (1998) 105-111.

[29] Y.T. Lei, Z.Y. Chen, X.Q. An, M.J. Huang, W.G. Shen, J. Chem. Eng. Data 55 (2010) 4154-4161.

[30] C.A. Tovar, E. Carballo, C.A. Cerdeirina, J.L. Legido, L. Romani, Int. J. Thermophys. 18 (1997) 761-777.

[31] H.L. Finke, M.E. Gross, G. Waddington, H.M. Huffman, JACS 76 (1954) 333-341.

[32] J.M. Pardo, C.A. Tovar, J. Troncoso, E. Carballo, L. Romani, Thermochim. Acta 433 (2005) 128-133.

[33] E. Wilhelm, A. Inglese, J.R. Quint, J.P.E. Grolier, J. Chem. Thermodyn. 14 (1982) 303-

308.

[34] N.S. Osborne, D.C. Ginnings, J. Res. Nat. Bur. Stand. 39 (1947) 453-477.

[35] B. Lagourette, Boned, C., Saintguirons, H., Xans, P., and Zhou, H., Meas. Sci. Technol. 3 (1992) 699-703.

[36] E.F. May, W.J. Tay, M. Nania, A. Aleji, S. Al-Ghafri, J.P.M. Trusler, Rev. Sci. Instrum. 85 (2014) 095111.

[37] W. Wagner, A. Pruss, J. Phys. Chem. Ref. Data 31 (2002) 387-535.

[38] D.O. Ortiz-Vega, A New Wide Range Equation Of State For Helium-4, Chem. Eng. (N.Y.), Texas A\&M University, 2013. 
[39] D.O. Ortiz-Vega, K.R. Hall, J.C. Holste, V.D. Arp, A.H. Harvey, E.W. Lemmon, J. Phys. Chem. Ref. Data (To be published) (2018).

[40] M.O. McLinden, J.D. Splett, J. Res. Natl. Inst. Stand. Technol. 113 (2008) 29-67.

[41] E.W. Lemmon, M.L. Huber, M.O. McLinden, NIST standard reference database 23: Reference fluid thermodynamic and transport properties - REFPROP, Version 9.1, National Institute of Standards and Technology Gaithersburg, 2013.

[42] C.-W. Lin, J.P.M. Trusler, J. Chem. Phys. 136 (2012).

[43] J.P.M. Trusler, Physical Acoustics and Metrology of Fluids, Adam Higler, Bristol, 1991.

[44] J.H. Dymond, R. Malhotra, Int. J. Thermophys. 9 (1988) 941-951.

[45] L.A. Davis, R.B. Gordon, J. Chem. Phys. 46 (1967) 2650-2660.

[46] T.F. Sun, C.A. Tenseldam, P.J. Kortbeek, N.J. Trappeniers, S.N. Biswas, Phys. Chem. Liq. 18 (1988) 107-116.

[47] F. Peleties, J.J. Segovia, J.P.M. Trusler, D. Vega-Maza, J. Chem. Thermodyn. 42 (2010) 631-639.

[48] J.P.M. Trusler, E.W. Lemmon, J. Chem. Thermodyn. 109 (2017) 61-70.

[49] M.J. Davila, J.P.M. Trusler, J. Chem. Thermodyn. 41 (2009) 35-45.

[50] C.-W. Lin, D. Ramjugernath, P. Reddy, J.P.M. Trusler, J. Chem. Eng. Data 57 (2012)

2568-2575. 


\title{
Density, Sound Speed and Derived Thermophysical Properties of $n$-Nonane at Temperatures Between (283.15 and 473.15) K and at Pressures up to $390 \mathrm{MPa}$
}

\author{
Weparn J Tay and J P Martin Trusler \\ Department of Chemical Engineering, Imperial College London, South Kensington Campus, \\ London SW7 2AZ
}

\section{SUPPLEMENTARY MATERIAL}

\section{Density of Methylbenzene}

Table S.1. Density $\rho$ for methylbenzene at temperatures $T$ and pressure $p .^{a}$

\begin{tabular}{|c|c|c|c|c|c|}
\hline$p / \mathrm{MPa}$ & $\rho /\left(\mathrm{kg} \cdot \mathrm{m}^{-3}\right)$ & $p / \mathrm{MPa}$ & $\rho /\left(\mathrm{kg} \cdot \mathrm{m}^{-3}\right)$ & $p / \mathrm{MPa}$ & $\rho /\left(\mathrm{kg} \cdot \mathrm{m}^{-3}\right)$ \\
\hline \multicolumn{6}{|c|}{$T=283.16 \mathrm{~K}$} \\
\hline 1.02 & 876.55 & 25.06 & 892.35 & 50.01 & 906.43 \\
\hline 5.01 & 879.36 & 30.04 & 895.27 & 55.06 & 909.09 \\
\hline 10.03 & 882.78 & 35.08 & 898.24 & 60.03 & 911.65 \\
\hline 15.05 & 886.11 & 40.06 & 901.05 & 65.05 & 914.15 \\
\hline 20.08 & 889.23 & 45.05 & 903.77 & & \\
\hline \multicolumn{6}{|c|}{$T=298.15 \mathrm{~K}$} \\
\hline 1.04 & 862.88 & 25.05 & 879.93 & 50.04 & 895.06 \\
\hline 5.04 & 865.93 & 30.06 & 883.14 & 55.04 & 897.86 \\
\hline 10.03 & 869.60 & 35.02 & 886.25 & 60.03 & 900.56 \\
\hline 15.05 & 873.21 & 40.04 & 889.26 & 65.02 & 903.20 \\
\hline 20.04 & 876.63 & 45.07 & 892.20 & & \\
\hline \multicolumn{6}{|c|}{$T=323.15 \mathrm{~K}$} \\
\hline 1.05 & 839.46 & 25.06 & 859.05 & 50.01 & 875.93 \\
\hline 5.05 & 843.04 & 30.08 & 862.69 & 55.01 & 879.01 \\
\hline 10.03 & 847.29 & 35.02 & 866.13 & 60.05 & 882.04 \\
\hline 15.01 & 851.38 & 40.01 & 869.52 & 65.03 & 884.91 \\
\hline 20.01 & 855.27 & 45.06 & 872.84 & & \\
\hline \multicolumn{6}{|c|}{$T=348.15 \mathrm{~K}$} \\
\hline 1.06 & 815.55 & 25.02 & 838.20 & 50.02 & 857.09 \\
\hline 5.07 & 819.75 & 30.08 & 842.30 & 55.06 & 860.49 \\
\hline 10.01 & 824.67 & 35.05 & 846.21 & 60.04 & 863.80 \\
\hline 15.03 & 829.43 & 40.05 & 849.97 & 65.07 & 867.00 \\
\hline 20.03 & 833.88 & 45.07 & 853.63 & & \\
\hline \multicolumn{6}{|c|}{$T=373.15 \mathrm{~K}$} \\
\hline 1.05 & 790.86 & 25.08 & 817.35 & 50.04 & 838.50 \\
\hline 5.02 & 795.87 & 30.07 & 821.93 & 55.04 & 842.23 \\
\hline 10.04 & 801.74 & 35.04 & 826.36 & 60.01 & 845.81 \\
\hline
\end{tabular}




\begin{tabular}{|c|c|c|c|c|c|}
\hline $\mathrm{p} / \mathrm{MPa}$ & $\rho /\left(\mathrm{kg} \cdot \mathrm{m}^{-3}\right)$ & $p / M P a$ & $\rho /\left(\mathrm{kg} \cdot \mathrm{m}^{-3}\right)$ & $\mathrm{p} / \mathrm{MPa}$ & $\rho /\left(\mathrm{kg} \cdot \mathrm{m}^{-3}\right)$ \\
\hline 15.04 & 807.26 & 40.04 & 830.59 & & \\
\hline 20.03 & 812.38 & 45.03 & 834.57 & & \\
\hline \multicolumn{6}{|c|}{$T=398.15 \mathrm{~K}$} \\
\hline 1.07 & 765.25 & 25.05 & 796.25 & 50.02 & 819.96 \\
\hline 5.03 & 771.26 & 30.04 & 801.49 & 55.04 & 824.15 \\
\hline 10.04 & 778.22 & 35.04 & 806.48 & 60.02 & 828.11 \\
\hline 15.08 & 784.74 & 40.02 & 811.17 & 65.08 & 831.99 \\
\hline 20.01 & 790.62 & 45.04 & 815.71 & & \\
\hline \multicolumn{6}{|c|}{$T=423.15 \mathrm{~K}$} \\
\hline 1.05 & 738.06 & 25.02 & 774.89 & 50.04 & 801.63 \\
\hline 5.03 & 745.45 & 30.02 & 780.87 & 55.06 & 806.24 \\
\hline 10.06 & 753.93 & 35.06 & 786.56 & 60.04 & 810.55 \\
\hline 15.03 & 761.54 & 40.02 & 791.82 & 65.05 & 814.77 \\
\hline 20.00 & 768.41 & 45.04 & 796.87 & & \\
\hline \multicolumn{6}{|c|}{$T=448.15 \mathrm{~K}$} \\
\hline 1.04 & 708.96 & 25.05 & 753.39 & 50.03 & 783.39 \\
\hline 5.07 & 718.48 & 30.04 & 760.21 & 55.06 & 788.44 \\
\hline 10.04 & 728.71 & 35.04 & 766.59 & 60.01 & 793.17 \\
\hline 15.07 & 737.86 & 40.02 & 772.48 & 65.06 & 797.83 \\
\hline 20.05 & 745.94 & 45.03 & 778.13 & & \\
\hline \multicolumn{6}{|c|}{$T=473.15 \mathrm{~K}$} \\
\hline 1.03 & 676.80 & 25.04 & 731.39 & 50.05 & 765.18 \\
\hline 5.05 & 689.34 & 30.01 & 739.18 & 55.02 & 770.69 \\
\hline 10.05 & 702.19 & 35.04 & 746.42 & 60.03 & 775.95 \\
\hline 15.06 & 713.20 & 40.05 & 753.09 & 65.02 & 780.92 \\
\hline 20.02 & 722.73 & 45.06 & 759.38 & & \\
\hline
\end{tabular}

a Expanded uncertainties are $U(T)=0.05 \mathrm{~K}, U(p)=0.03 \mathrm{MPa}$ and $U_{\mathrm{c}}(\rho)=0.6 \mathrm{~kg} \cdot \mathrm{m}^{-3}$ with a coverage factor $k=2$.

\section{Thermodynamic Integration Sensitivity Analysis}

As described in the main paper, the effects of cosinusoidal temperature-dependent and pressure-dependent relative perturbations to the speed of sound surface were considered. These perturbations were defined such that the perturbed value $c_{\text {pert }}$ of the speed of sound $c$ was given by

$$
c_{\text {pert }}=c\left[1+A \cdot \cos \left(k_{1} T\right) \cos \left(k_{2} p\right)\right]
$$

where, $A$ is an amplitude and $k_{1}$ and $k_{2}$ determine the wavelengths of the perturbation in temperature and pressure. The amplitudes $A$ considered was $0.1 \%$. Figs. S1 to S4 show the changes in the derived values of density $\rho$, isobaric specific heat capacity $c_{p}$, isobaric expansivity $\alpha_{p}$ and isothermal compressibility $\beta_{T}$ associated with this perturbation for several combinations of $k_{1}$ and $k_{2}$. 

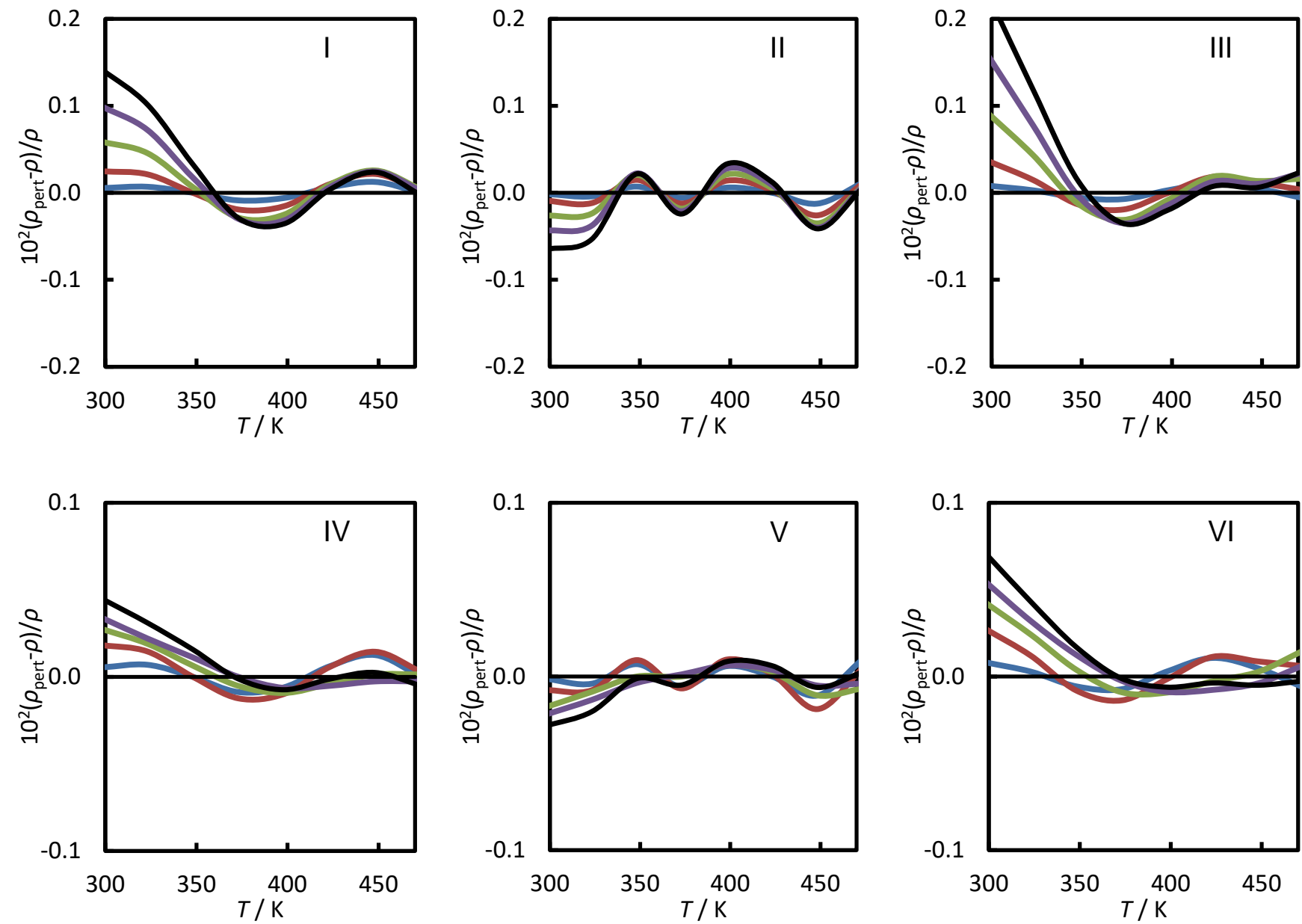

Fig. S1. Change in density $\rho$ arising from cosinusodal perturbations to the speed of sound with amplitude 0.1 \%: (I) $k_{1}=0.05, k_{2}=0$; (II) $k_{1}=0.1, k_{2}=0$; (III) $k_{1}=0.2, k_{2}=0$; (IV) $k_{1}=0.05, k_{2}=0.02$; (V) $k_{1}=0.1, k_{2}=0.02$; (VI) $k_{1}=0.2, k_{2}=0.02$. Curves: (blue), $p=30 \mathrm{MPa}$; (red), $p=90 \mathrm{MPa}$; (green), $p=180 \mathrm{MPa}$; (purple), $p=$ $270 \mathrm{MPa}$; (black) $p=360 \mathrm{MPa}$. 

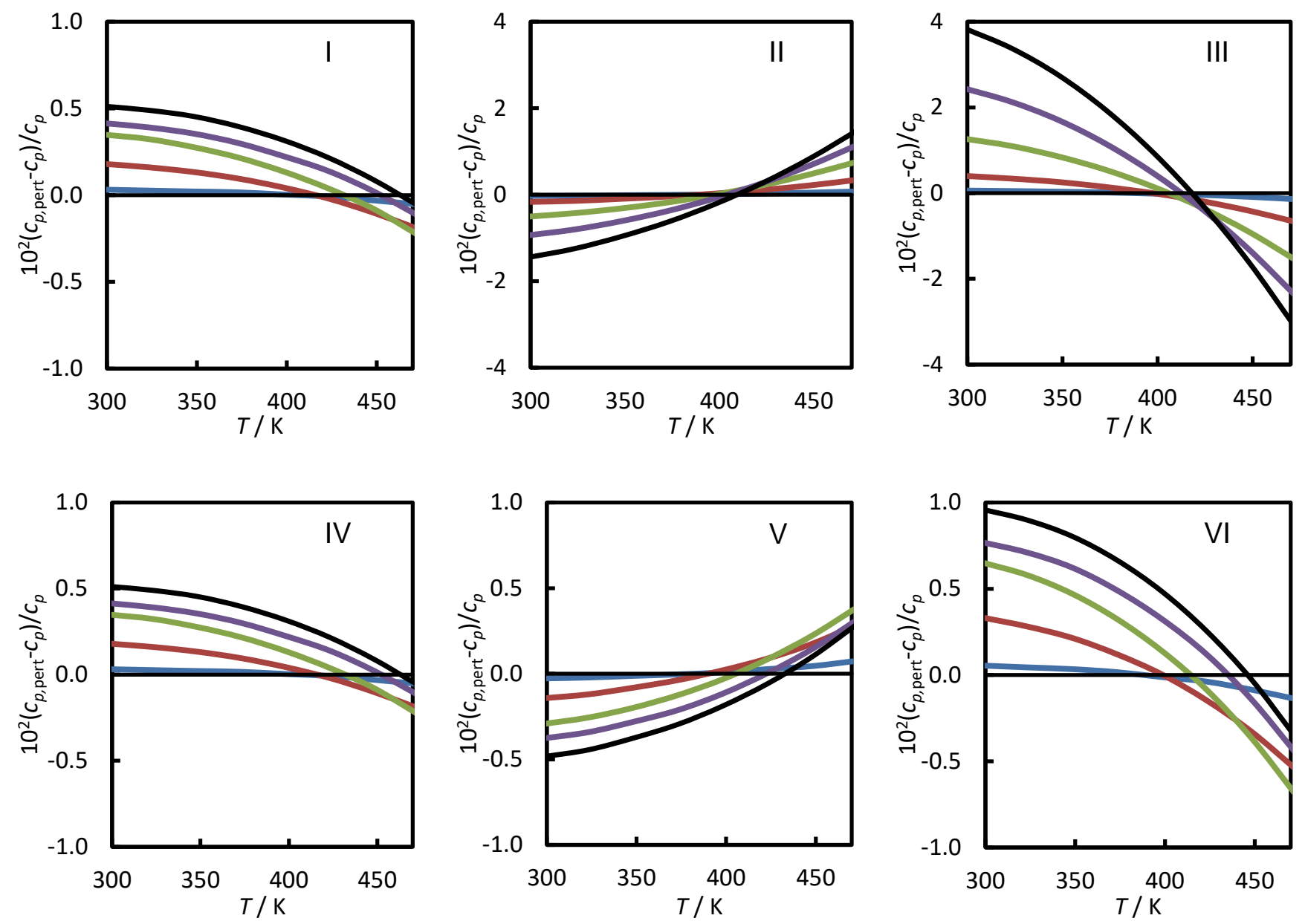

Fig. S2. Change in isobaric specific heat capacity $c_{p}$ arising from cosinusodal perturbations to the speed of sound with amplitude $0.1 \%$ : (I) $k_{1}=0.05, k_{2}=0$; (II) $k_{1}=0.1, k_{2}=0$; (III) $k_{1}=0.2, k_{2}=0$; (IV) $k_{1}=0.05, k_{2}=$ 0.02; (V) $k_{1}=0.1, k_{2}=0.02 ;(\mathrm{VI}) k_{1}=0.2, k_{2}=0.02$. Curves: (blue), $p=30 \mathrm{MPa}$; (red), $p=90 \mathrm{MPa}$; (green), $p$ $=180 \mathrm{MPa}$; (purple), $p=270 \mathrm{MPa}$; (black) $p=360 \mathrm{MPa}$. 

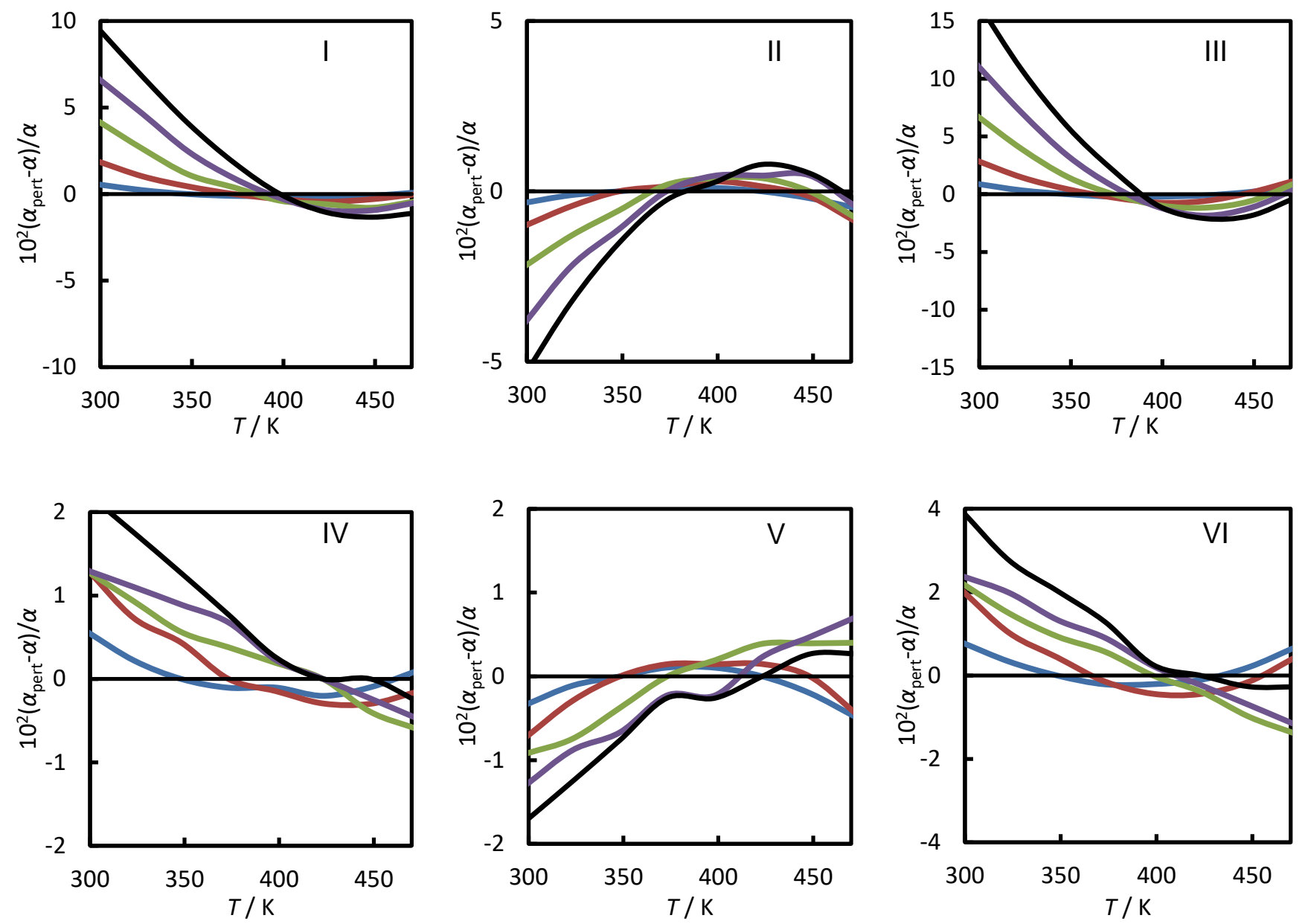

Fig. S3. Change in isobaric expansivity $\alpha_{p}$ arising from cosinusodal perturbations to the speed of sound with amplitude $0.1 \%$ : (I) $k_{1}=0.05, k_{2}=0$; (II) $k_{1}=0.1, k_{2}=0$; (III) $k_{1}=0.2, k_{2}=0$; (IV) $k_{1}=0.05, k_{2}=0.02$; (V) $k_{1}=$ $0.1, k_{2}=0.02 ;(\mathrm{VI}) k_{1}=0.2, k_{2}=0.02$. Curves: (blue), $p=30 \mathrm{MPa}$; (red), $p=90 \mathrm{MPa}$; (green), $p=180 \mathrm{MPa}$; (purple), $p=270 \mathrm{MPa}$; (black) $p=360 \mathrm{MPa}$. 

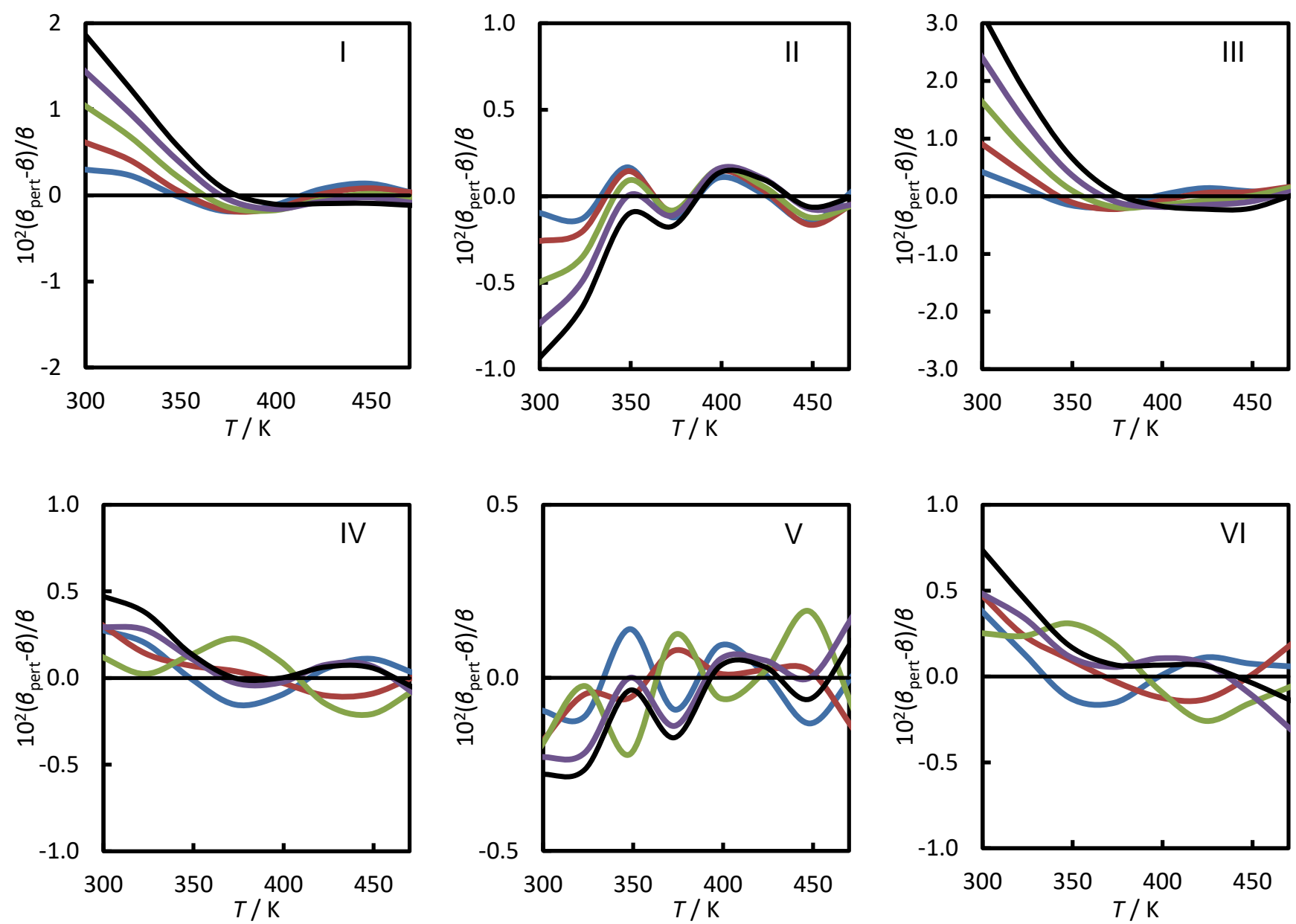

Fig. S4. Change in isothermal compressibility $\beta_{T}$ arising from cosinusodal perturbations to the speed of sound with amplitude $0.1 \%$ : (I) $k_{1}=0.05, k_{2}=0$; (II) $k_{1}=0.1, k_{2}=0$; (III) $k_{1}=0.2, k_{2}=0$; (IV) $k_{1}=0.05, k_{2}=0.02$; (V) $k_{1}=0.1, k_{2}=0.02 ;(\mathrm{VI}) k_{1}=0.2, k_{2}=0.02$. Curves: (blue), $p=30 \mathrm{MPa}$; (red), $p=90 \mathrm{MPa}$; (green), $p=180$ $\mathrm{MPa}$; (purple), $p=270 \mathrm{MPa}$; (black) $p=360 \mathrm{MPa}$. 